\title{
Lycopene alleviates hepatic ischemia reperfusion injury via the Nrf2/HO-1 pathway mediated NLRP3 inflammasome inhibition in Kupffer cells
}

\author{
Rong Xue ${ }^{1,2 \#}$, Jiannan Qiu ${ }^{2,3 \#}$, Song Wei, ${ }^{1,3 \#}$, Mu Liu ${ }^{2,3 \#}$, Qi Wang ${ }^{1,3}$, Peng Wang ${ }^{2,3}$, Bowen Sha ${ }^{2,3}$, \\ Hao Wang ${ }^{2,3}$, Yong $\mathrm{Shi}^{2,3}$, Jinren $\mathrm{Zhou}^{2,3}$, Jianhua $\mathrm{Rao}^{2}$, Ling $\mathrm{Lu}^{1,2,3,4,5,6}$
}

${ }^{1}$ School of Medicine, Southeast University, Nanjing, China; ${ }^{2}$ Hepatobiliary Center of The First Affiliated Hospital, Nanjing Medical University, Nanjing, China \& Research Unit of Liver Transplantation and Transplant Immunology, Chinese Academy of Medical Sciences, Nanjing, China; ${ }^{3}$ The Affiliated Cancer Hospital (Jiangsu Cancer Hospital), Nanjing Medical University, Nanjing, China; ${ }^{4}$ State Key Laboratory of Reproductive Medicine, Nanjing Medical University, Nanjing, China; ${ }^{5}$ Jiangsu Collaborative Innovation Center of Biomedical Functional Materials, College of Chemistry and Materials Science, Nanjing Normal University, Nanjing, China; J Jiangsu Key Lab of Cancer Biomarkers, Prevention and Treatment, Collaborative Innovation Center for Personalized Cancer Medicine, Nanjing Medical University, Nanjing, China

Contributions: (I) Conception and design: L Lu, J Qiu, R Xue; (II) Administrative support: L Lu, J Rao; (III) Provision of study materials or patients: None; (IV) Collection and assembly of data: R Xue, J Qiu, S Wei, M Liu; (V) Data analysis and interpretation: R Xue, J Qiu, S Wei, Q Wang, M Liu, P Wang, B Sha, H Wang, Y Shi, J Zhou; (VI) Manuscript writing: All authors; (VII) Final approval of manuscript: All authors.

\#These authors contributed equally to this work.

Correspondence to: Ling Lu. School of Medicine, Southeast University, 87 Ding Jia Qiao Road, Nanjing, China. Email: lvling@njmu.edu.cn; Jianhua Rao. Hepatobiliary Center of The First Affiliated Hospital and Research Unit of Liver Transplantation and Transplant Immunology, Chinese Academy of Medical Sciences, 300 Guangzhou Road, Nanjing, China. Email: raojh@njmu.edu.cn.

Background: Lycopene is a naturally occurring carotenoid found in many fruits and vegetables, which has antioxidant effects. Although lycopene's protective effect has been observed on ischemia reperfusion (IR) injury in different organs, the effect of lycopene on Kupffer cells (KCs) has not been clearly elucidated in IRinduced acute hepatic inflammatory injury.

Methods: Mice were administered with either olive oil $(10 \mathrm{~mL} / \mathrm{kg}$ body weight $)$ as the control or lycopene ( $20 \mathrm{mg} / \mathrm{kg}$ body weight) by gavage for 2 weeks before undergoing hepatic IR injury.

Results: In this study, we observed that the levels of aspartate aminotransferases (AST), alanine aminotransferase (ALT), and the percentages of hepatocellular apoptosis in mice pretreated with lycopene were significantly lower than control mice. Lycopene inhibited F4/80+ macrophage and Ly6G+ neutrophil accumulation, which further decreased the levels of tumor necrosis factor- $\alpha$ (TNF- $\alpha$ ), interleukin-1 $\beta$ (IL-1 $\beta$ ), and interleukin 6 (IL-6). Interestingly, lycopene induced increased autophagy in KCs, which was evidenced by elevated autophagosomes and the increased protein level of LC3B. In these KCs, lycopene-induced upregulation of autophagy inhibited NOD-like receptor family pyrin domain-containing 3 protein (NLRP3) inflammasome activation, which was demonstrated by the reduced mRNA and protein levels of NLRP3, cleaved caspase-1, an apoptosis-associated speck-like protein containing a caspase recruitment domain (ASC), and IL-1 $\beta$. Furthermore, 3-methyladenine, an autophagy inhibitor, abolished lycopene's inhibitory effect on the NLRP3 inflammasome in KCs, which led to increased hepatic IR injury. Intriguingly, we identified that the protein levels of nuclear factor erythroid 2-related factor 2 (Nrf2) and heme oxygenase 1 (HO-1) were elevated in KCs isolated from IR-stressed mice pretreated with lycopene. Nrf2-siRNA or HO-1-siRNA could block the autophagy activation enhanced by lycopene in KCs, resulting in the activation of the NLRP3 inflammasome and aggravated hepatic IR injury.

Conclusions: Our findings demonstrated that lycopene promoted Nrf2/HO-1 pathway activation and further suppressed the NLRP3 inflammasome via enhancing KC autophagy, which alleviated hepatic IR injury. 
Keywords: Lycopene; hepatic ischemia reperfusion injury (hepatic IR injury); Kupffer cells (KCs); NLRP3 inflammasome; autophagy

Submitted Oct 23, 2020. Accepted for publication Jan 08, 2021.

doi: 10.21037/atm-20-7084

View this article at: http://dx.doi.org/10.21037/atm-20-7084

\section{Introduction}

Hepatic ischemia reperfusion (IR) injury remains the leading cause of acute hepatic dysfunction and failure after undergoing trauma, hepatectomy, and liver transplantation, which results in a poor prognosis for these patients (1). The pathological process of hepatic IR injury is characterized by mitochondrial dysfunction, cellular calcium overload, overproduction of reactive oxygen species (ROS), and overactivation of inflammatory cells $(2,3)$. It has been reported that Kupffer cells (KCs) are integrally involved in the pathogenesis of hepatic IR injury by regulating acute immune responses $(4,5)$. Increasing evidence suggests that quiescent KCs play a critical role in maintaining hepatic immune homeostasis; however, this can be disrupted by activated KCs through the production of ROS and the secretion of pro-inflammatory factors and chemokines, which recruit multiple immune cells to hepatic tissue (6). Furthermore, a growing number of studies have demonstrated that the activation of the NLRP3 inflammasome in KCs is closely involved in hepatic IR injury $(7,8)$. The NLRP3 inflammasome made up of the NOD-like receptor NLRP3, the enzyme caspase-1, and the adaptor molecule ASC (9), generates a mass of proinflammatory cytokines in IR injury, such as IL- $1 \beta$ and IL-18 $(10,11)$. As a cytoprotective process, autophagy is featured by conserved self-eating and contributes to the degradation of intracellular components, including dysfunctional organelles, macromolecular complexes, long-lived cytoplasmic proteins, and foreign bodies (12). It has been well reported in many studies that autophagy plays a vital role in the pathological processes of hepatic diseases, including tumors (13), viral hepatitis (14), liver IR injury (15), and steatohepatitis (16), amongst others. Recent studies demonstrated that cerebral and myocardial IR injury was attenuated by enhanced autophagy through suppressing NLRP3 inflammasome activation $(17,18)$. Nevertheless, the relationship between the NLRP3 inflammasome and autophagy in hepatic IR injury has rarely been reported.
Nuclear factor erythroid 2-related factor 2 (Nrf2), well known as the central regulator of cellular defense, maintains cellular redox homeostasis $(19,20)$. Nrf2 contains $7 \mathrm{Neh}$ (Nrf2-ECH homology) domains and is localized in the cytoplasm under physiological conditions. Kelchlike erythroid-associated protein 1 (Keap1) maintains Nrf2 homeostasis through the ubiquitination and proteasome pathway $(19,21)$. However, Nrf2 is translocated into the nucleus and combines with the antioxidant response elements (AREs) of multiple protective genes under stress conditions, contributing to cytoprotective and antioxidative effects (22). Emerging evidence indicates that $\mathrm{Nrf2}$ is involved in liver diseases via regulating autophagy (23). Many studies have reported that the Nrf2/HO-1 pathway plays a critical role in regulating hepatic IR injury. Our previous data showed that ATF3-mediated Nrf2/HO-1 signaling played an important role in regulating TLR4-driven inflammatory responses in IR-stressed livers (24). Recent results further indicated that the Nrf2/HO-1 pathway activation could improve hepatic IR injury in mice (25-27). However, no studies have reported that $\mathrm{Nrf} 2 / \mathrm{HO}-1$ signaling can regulate autophagy in KCs in hepatic IR injury.

Lycopene is one dietary carotenoid found in fruits and vegetables (28), which possesses pharmacological effects including antioxidant (29), anti-inflammatory (30), and anticancer effects (31), and regulates lipid metabolism (32). Numerous studies have confirmed that lycopene plays an essential role in improving IR injury of the brain (33), heart (34), liver (35), and kidney (36). However, the underlying mechanisms of how lycopene alleviates hepatic IR injury remain to be elucidated.

In the present study, we demonstrated that lycopene inhibits the activation of the NLRP3 inflammasome in KCs via activating the Nrf2/HO-1 pathway and enhancing autophagy, which attenuates hepatic damage and acute immune responses in a hepatic IR mouse model.

We present the following article following the ARRIVE reporting checklist (available at http://dx.doi.org/10.21037/ atm-20-7084). 


\section{Methods}

\section{Animals}

The animal experiments in this study were approved by the Ethics Committee of Nanjing Medical University (Approval No.: IACUC-1912015), in compliance with the Nanjing Medical University guidelines for the care and use of animals. Male wild type C57BL/6 mice (8-10 weeks old) were purchased from the Department of Laboratory Animal Center of Nanjing Medical University. All mice were housed in a specific pathogen-free facility following institutional guidelines.

\section{Hepatic IR injury model and treatment}

The procedure for establishing the mouse hepatic IR injury model was the same as described in a previous study (37). In brief, the mice $(n=6)$ were firstly anesthetized; then, a ventral linea alba incision was made. The partial portal vein and hepatic artery supplying the middle and left lateral liver lobes were occluded with an atraumatic vascular clip for 90 min to cause ischemia in $70 \%$ of liver tissue, followed by $6 \mathrm{~h}$ reperfusion by removing the clip. The same procedures were performed in the sham group without vascular clamping. Mice were administered with either olive oil $(10 \mathrm{~mL} / \mathrm{kg}$ body weight) as a control or lycopene ( $20 \mathrm{mg} / \mathrm{kg}$ body weight) by gavage for 2 weeks before undergoing IR injury. The dose of lycopene was based on Boeira et al.'s paper (38). Depending on whether they were pretreated with lycopene, mice were divided into the Lycopene(-) and Lycopene(+) group. The autophagy inhibitor 3-methyladenine (3MA, $30 \mathrm{mg} / \mathrm{kg}$ ) (Sigma-Aldrich, St. Louis, MO, USA) was administered intraperitoneally $60 \mathrm{~min}$ before establishing the IR model, and the same volume of PBS was administered intraperitoneally into the control mice. Some mice were injected via the tail vein with Nrf2-siRNA, HO-1-siRNA, or scrambled control siRNA (SCR-siRNA) $(2 \mathrm{mg} / \mathrm{kg}$ ) (Santa Cruz, CA, USA) mixed with mannose-conjugated polymers (Polyplus-transfection, Illkirch, France) at ratios based on the manufacturer's instructions $4 \mathrm{~h}$ before the establishment of the hepatic IR model as previously described (39).

\section{Biochemical measurements}

Blood samples, obtained when mice were sacrificed, were centrifuged at $7,000 \mathrm{rpm}$ for $10 \mathrm{~min}$ at $4{ }^{\circ} \mathrm{C}$ to collect the serum. The levels of serum alanine aminotransferase (sALT) and aspartate aminotransferase (sAST) were measured by an automatic chemical analyzer (Olympus Company, Tokyo, Japan).

\section{Histopathology, immunohistochemical staining, immunofluorescence staining, and TUNEL staining}

Hepatic tissue samples, obtained when mice were sacrificed, were fixed in formaldehyde solution for approximately 3 days, then embedded in paraffin-based on standard procedures. All paraffin blocks were cut into $4 \mu \mathrm{m}$ slices and stained with hematoxylin and eosin (HE), then observed through a light microscope for analyzing hepatic histopathology. Suzuki scores, consisting of 3 aspects (hepatocyte necrosis, sinusoidal congestion, and ballooning degeneration), were rated on a scale of 1 to 4 , as defined by Suzuki et al. (40). Hepatic macrophages and neutrophils were detected by immunohistochemical (IHC) staining through incubation with the primary rat anti-mouse F4/80 and Ly6G mAbs (Cell Signaling Technology, MA, USA). Positive cells were counted blindly in at least 3 fields per section $(\times 200)$. The expression of NLRP3 and LC3B in KCs were detected by immunofluorescence staining using rabbit anti-mouse NLRP3 $\mathrm{mAb}$ and anti-mouse LC3B mAb (Cell Signaling Technology, MA, USA). According to the manufacturer's protocols, the secondary, biotinylated goat anti-rat IgG (Vector, Burlingame, CA, USA) was incubated with immunoperoxidase (ABC Kit, Vector). DAPI was utilized for nuclear counterstaining. To detect DNA fragmentation as a characteristic of apoptosis in formalinfixed paraffin-embedded tissue slices, we performed TUNEL staining using the Klenow-FragEL DNA Fragmentation Detection Kit (Roche, Basel, Switzerland) according to the manufacturer's instructions. Ten fields from each slide were selected randomly for examination $(\times 400)$.

\section{Myeloperoxidase assay}

Detection of hepatic myeloperoxidase (MPO) activity was performed according to the manufacturer's protocols. Detailed processes have been described previously (4).

\section{Transmission electron microscopy analysis}

The autophagic vacuoles in KCs were detected by transmission electron microscopy according to the manufacturer's instructions. In short, the culture media was discarded, then the trypsin was added into the culture 
dish to digest KCs adequately for $3 \mathrm{~min}$. Media was used to stop digestion, and KCs were collected into $15 \mathrm{~mL}$ centrifugal tubes with a pasteur pipette. Cell suspensions were centrifuged at 2,500 rpm for $2 \mathrm{~min}$. The supernatant was then discarded, and cell pellets were fixed with $2.5 \%$ glutaraldehyde at room temperature in the dark for $30 \mathrm{~min}$. Centrifugal tubes were then transferred to the refrigerator for preservation at $4{ }^{\circ} \mathrm{C}$. The embedded materials were cut into ultrathin sections. The sections were further stained with $0.3 \%$ lead citrate. To assess autophagic vacuoles in KCs, 10 micrographs from each group were selected randomly for examination $(\times 1,200$ or $\times 5,000)$.

\section{Cell isolation and culture}

The process of isolating KCs from the liver has been previously described (41). In short, we performed portal vein catheterization and perfused the livers with preheated $\left(37^{\circ} \mathrm{C}\right)$ Hanks' balanced salt solution (HBSS), followed by collagenase IV.

After sufficient perfusion, the liver was transferred to a culture dish, dissected, and ground softly, then filtered with a $70 \mathrm{~mm}$ nylon mesh cell strainer. The centrifugal tube containing whole hepatic cells was centrifuged at $50 \mathrm{~g}$ for 2 min 3 times. We collected the supernatant, which contained the nonparenchymal cells (NPCs). Centrifugation at $800 \mathrm{~g}$ for 5 min was performed to obtain the NPCs in the supernatant. The NPC suspension underwent a $50 \% / 25 \%$ two-step percoll gradient centrifugation at $1,800 \times \mathrm{g}$ at $4{ }^{\circ} \mathrm{C}$ for $15 \mathrm{~min}$. We collected the middle layer, which was comprised of KCs, and allowed them to attach to a culture dish with complete media.

We discarded the culture media to remove the nonadherent cells so that the adherent cells were KCs. In vitro, the isolated $\mathrm{KCs}$ were cultured with complete media for $6 \mathrm{~h}$, then KCs and the supernatants were obtained for subsequent research.

\section{Protein extraction and western blotting}

We used the Whole Protein Extraction Kit (KeyGEN BioTECH) to extract total protein from tissues and cells. Additionally, we used the Nuclear and Cytoplasmic Protein Extraction Kit (KeyGEN BioTECH) to extract nuclear and cytoplasmic proteins from KCs based on the manufacturer's protocols. All protein lysates were separated by standard SDS-PAGE and then transferred onto polyvinylidene fluoride (PVDF) membranes. The antibodies used were as follows: Nrf2 (Abcam), Bcl2, Bcl-xL, NLRP3, cleaved caspase-1, pro-caspase-1, ASC, IL-1 $\beta$, pro-IL-1 $\beta$, LC3B, $\mathrm{p} 62$, HO-1, $\beta$-actin, and horseradish peroxidase (HRP)conjugated anti-rabbit IgG antibodies (Cell Signaling Technology).

\section{$R N A$ extraction and $q R T-P C R$}

We utilized the EasyPure RNA Kit (Ruijie Biotech) to extract total RNA from tissues and cell samples according to the manufacturer's protocols. In short, tissues or cells were mixed with the lysis buffer. After efficient lysis was confirmed, the lysis solution was collected and vortexed for $10 \mathrm{sec}$, then an equal volume of absolute ethyl alcohol was added and mixed. The lysis solution was transferred into an RNA column, which was placed in a centrifugal tube. The tube was centrifuged at $4,000 \times \mathrm{g}$ for $1 \mathrm{~min}$ at $4{ }^{\circ} \mathrm{C}$ and washed with $500 \mu \mathrm{L}$ wash buffer followed by centrifugation at $12,000 \times \mathrm{g}$ for $1 \mathrm{~min}$. Subsequently, the RNA column was transferred into a new and RNase-free tube and air-dried for $2 \mathrm{~min}$. We added $20 \mu \mathrm{L}$ elution buffer into the center of the RNA column and waited for $2 \mathrm{~min}$. Subsequently, the tube was centrifuged at $12,000 \times \mathrm{g}$ for $1 \mathrm{~min}$, and the extracted RNA was stored at $-80{ }^{\circ} \mathrm{C}$ before further experiments. For cDNA synthesis, a $20 \mu \mathrm{L} /$ well reaction mix was composed of $1 \mu \mathrm{g}$ total RNA, $4 \mu \mathrm{L} 5 \times$ HiScript IIqRT SuperMix (Vazyme Biotech Co., Ltd), and RNase-free $\mathrm{ddH}_{2} \mathrm{O}$.

We performed qRT-PCR with the ChamQTM Universal SYBR qPCR Master Mix (Vazyme Biotech Co., Ltd.) based on the standard protocols. Primer sequences used in this study for PCR amplification are listed in Figure S1.

\section{ELISA}

In this study, we obtained mouse serum and culture supernatants of KCs to analyze the levels of cytokines. According to the manufacturer, the levels of TNF- $\alpha$, IL- $1 \beta$, and IL-18 in mouse serum and culture supernatants of KCs were detected using ELISA kits (eBiosciences, San Diego, CA, USA)'s instructions.

\section{Caspase-3 activity assay}

Caspase-3 relative activity in hepatic tissue was detected with a caspase-3 assay kit (Calbiochem, La Jolla, CA, USA) according to the manufacturer's protocols. 


\section{Statistical analysis}

All data in this study are presented as mean \pm SD. We used SPSS software ver. 20.0 and GraphPad Prism 8.0 to analyze the data and calculate $P$ values. Statistical differences between multiple groups were evaluated using the Student's $t$-test or variance analysis (ANOVA). All experiments were repeated at least 3 times, and the differences were considered statistically significant when $\mathrm{P}$ value $<0.05$.

\section{Results}

\section{Lycopene alleviates IR-induced hepatocellular damage and apoptosis}

It is well known that lycopene possesses anti-inflammatory and antioxidative effects, which protects organs from various injuries. Hence, we further set out to demonstrate whether lycopene can improve hepatic IR injury in mice. We pretreated mice with lycopene at a dose of $20 \mathrm{mg} / \mathrm{kg}$ body weight or olive oil at a dose of $10 \mathrm{~mL} / \mathrm{kg}$ bodyweight for 2 weeks $(n=6)$. We found that serum ALT and AST levels in the group pretreated with lycopene were less than the control group pretreated with olive oil (Figure 1A). Compared to the control group, mice who received lycopene displayed wellpreserved hepatic architecture that was associated with less sinusoidal congestion, intracellular edema, and necrosis, which resulted in decreased total histopathological Suzuki score (Figure 1B,C).

Furthermore, lycopene markedly decreased serum MPO levels, which suggested that neutrophil activity or its cell numbers might have been reduced by lycopene (Figure 1D). To determine whether lycopene influences hepatic apoptosis induced by IR injury, hepatocellular apoptosis/necrosis in ischemic liver tissue was assessed by TUNEL staining and caspase- 3 activity assays. The TUNEL staining results showed that lycopene improved hepatic apoptosis, evidenced by fewer TUNEL positive cells (Figure 1E,F). Consistent with the TUNEL staining results, caspase-3 activity was decreased after lycopene pretreatment (Figure 1G). To further verify the above results, we used western blotting to detect the expression of the anti-apoptotic proteins $\mathrm{Bcl}-2$ and $\mathrm{Bcl}-\mathrm{xL}$ in tissue. We found that lycopene pretreatment significantly inhibited their expression induced by IR (Figure 1H). These results indicated that lycopene could alleviate IR-induced liver injury.

\section{Lycopene attenuates IR-induced acute liver inflammatory} responses

IR can induce acute liver inflammatory responses via activating KCs, recruiting circulating macrophages and neutrophils, or enhancing the production of proinflammatory factors. Our data showed that lycopene decreased serum MPO levels after reperfusion (Figure 1D). To further explore the relationship between the occurrence of acute inflammatory responses and lycopene in hepatic IR, F4/80+ macrophages and $\mathrm{Ly} 6 \mathrm{G}+$ neutrophils were assessed by IHC staining. As shown in Figure $2 A, B, C, D$, lycopene markedly decreased the accumulation of F4/80+ macrophages and Ly6G+ neutrophils in IR-stressed liver tissue. The lower levels of serum and mRNA TNF- $\alpha$ and IL-1 $\beta$ in mice that received lycopene pretreatment compared to the control group further demonstrated that lycopene could attenuate IR-induced acute liver inflammatory responses, which was also associated with reduced IL-6 and greater IL-10 expression (Figure 2E,F).

\section{Lycopene inbibits NLRP3 inflammasome activation in KCs via enhancing autophagy during bepatic IR injury}

Autophagic activity and NLRP3 inflammasome activation in KCs play a critical role in the level of acute liver inflammatory responses during IR injury. We started to assess the autophagic level in KCs isolated from mice pretreated with lycopene or olive oil 6 hours after reperfusion. Microtubule-associated proteins 1A/1B light chain 3B (LC3B) are the most widely used autophagosomes marker. The p62 protein is a classical receptor of autophagy and recognizes toxic cellular waste, and lack of autophagy leads to an accumulation of p62. We assessed LC3B and p62 protein expression in KCs by western blotting, which showed that lycopene pretreatment increased LC3B expression and decreased p62 expression after reperfusion (Figure 3A). The immunofluorescence staining of LC3B confirmed these results (Figure 3B).

Furthermore, the transmission electron microscopy results demonstrated that the number of autophagosomes was greater in the lycopene pretreatment group compared to the control group (Figure 3C). As shown in Figure 3D, the relative mRNA levels of NLRP3 and IL-1 $\beta$ were reduced significantly in KCs isolated from IR-stressed livers in the lycopene pretreatment group. As expected, the culture supernatants of KCs isolated from IR-stressed livers in the 
A

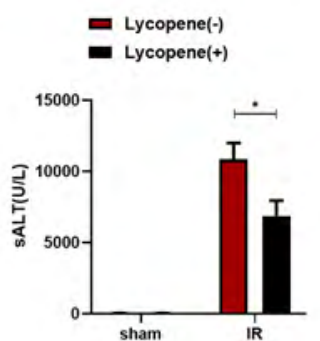

C

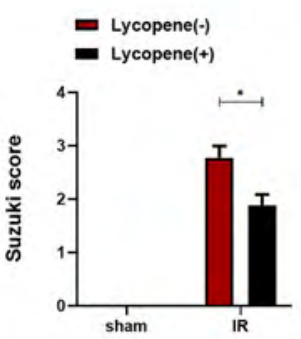

E
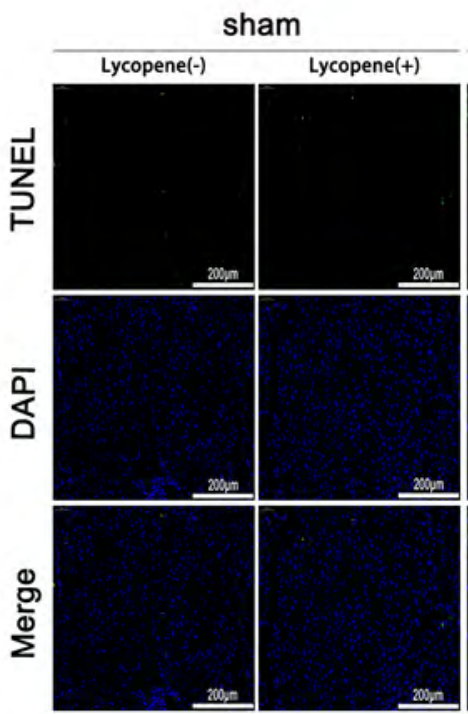

Dycopene(-)

- Lycopene(+)

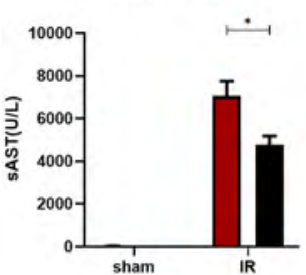

D

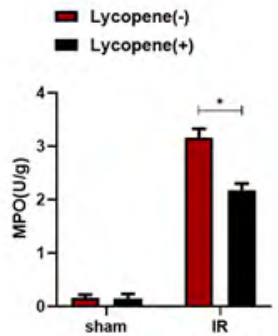

B
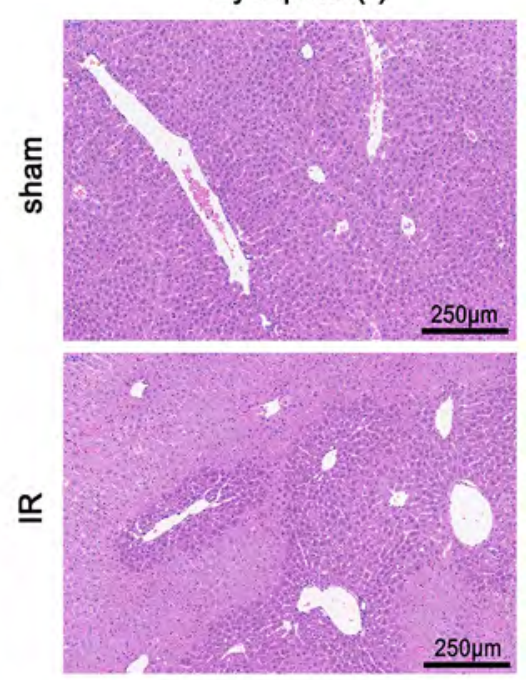

F
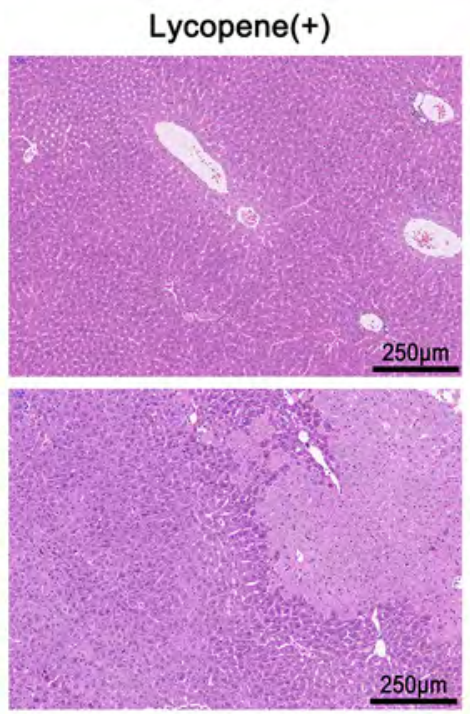

G

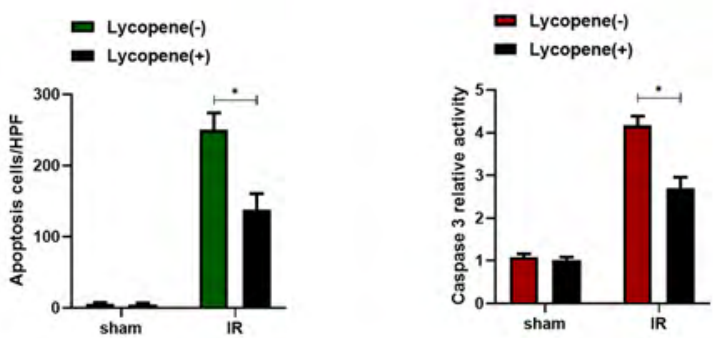

$\mathrm{H}$
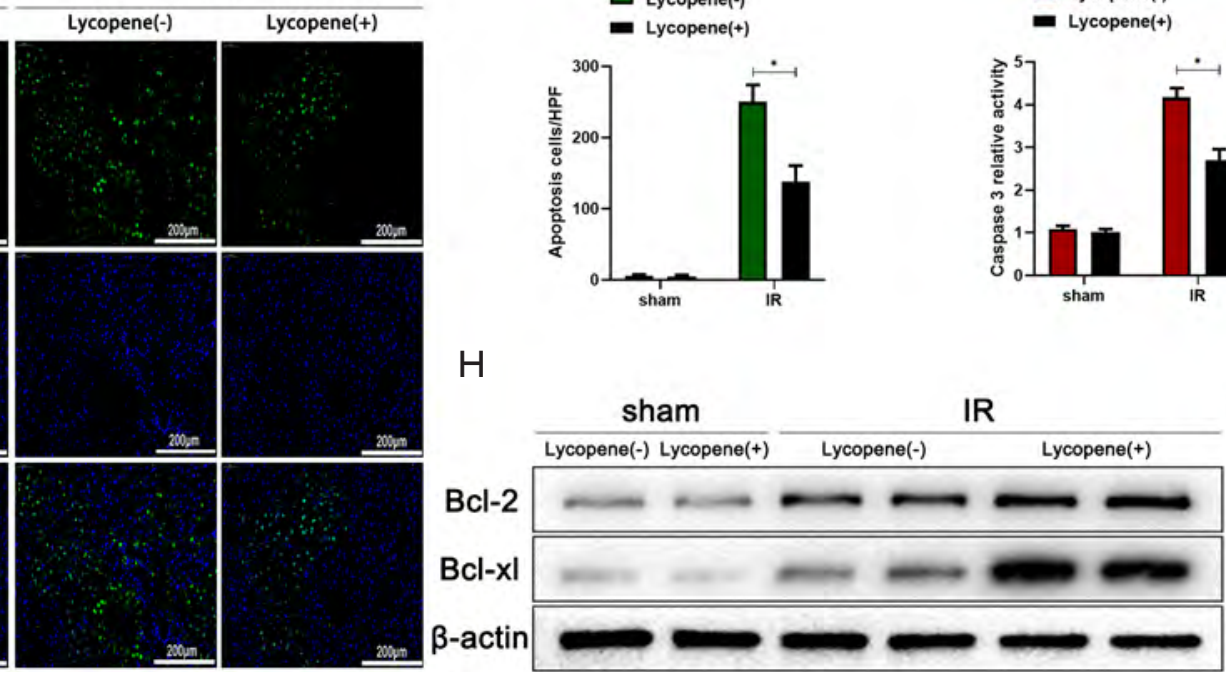

$\mathrm{kDa}$

26

30

Figure 1 Lycopene alleviates IR-induced acute liver injury. (A) Serum ALT and AST in mice from different groups who were subjected to sham operation or $70 \%$ hepatic ischemia for $90 \mathrm{~min}$, followed by $6 \mathrm{~h}$ of reperfusion ( $\mathrm{n}=6 \mathrm{mice} / \mathrm{group}$ ). (B) Representative hematoxylin and eosin (H\&E) staining of liver tissue in different groups (scale bars, $250 \mu \mathrm{m})$. (C,D) The average Suzuki scores and liver myeloperoxidase (MPO) activities in the different groups. (E,F) TUNEL staining (scale bars, $200 \mu \mathrm{m}$ ) and the relative ratios of TUNEL-positive cells across different groups. (G) Relative caspase-3 activity was evaluated from total lysates of lobes from IR mice or sham mice. (H) Western blot analysis of the protein levels of $\mathrm{Bcl}-2, \mathrm{Bcl}-\mathrm{xL}$, and $\beta$-actin for different groups. All data represent the mean $\pm \mathrm{SD},{ }^{*} \mathrm{P}<0.05$. ALT, alanine aminotransferase; AST, aspartate aminotransferases.

lycopene pretreatment group were characterized by lower levels of IL-1 $\beta$ and IL-18 (Figure 3E).

Furthermore, the western blotting results showed decreased protein levels of NLRP3, cleaved caspase-1, ASC,
IL-1 $\beta$, and pro-IL-1 $\beta$ in KCs isolated from IR-stressed mice in the lycopene pretreatment group (Figure $3 F$ ). The immunofluorescence staining results displayed that the intensity of NLRP3 was reduced in KCs isolated from IR- 


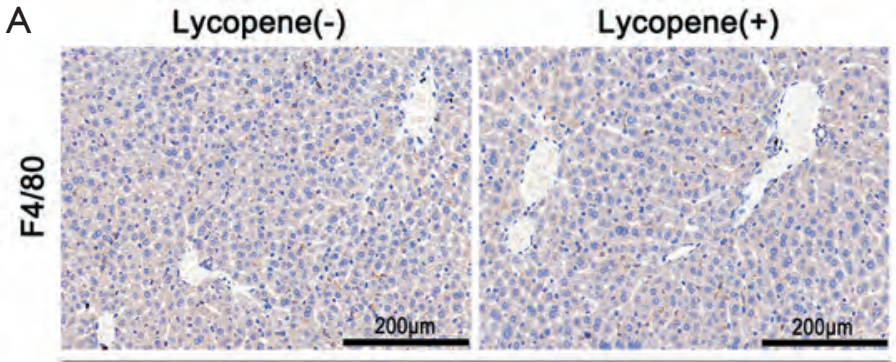

sham

B

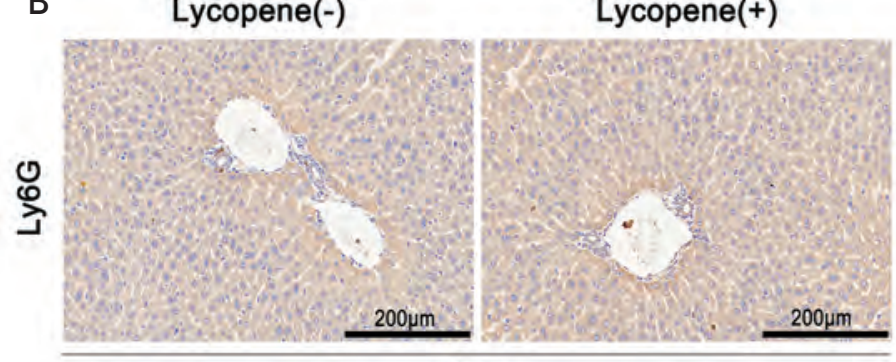

sham

C

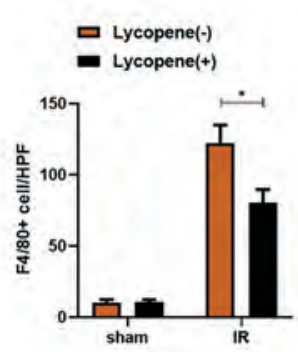

F

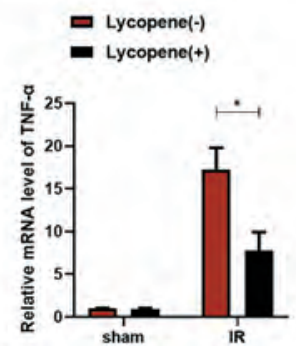

D
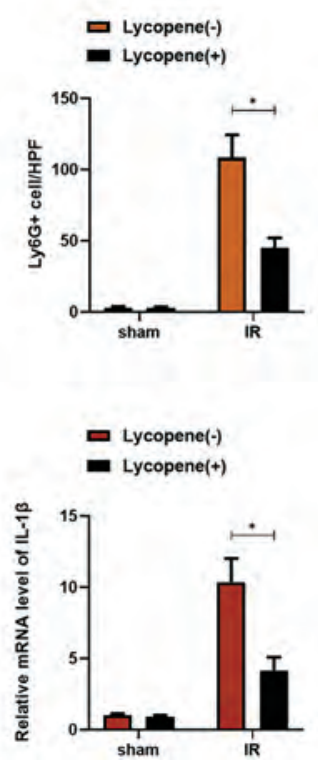
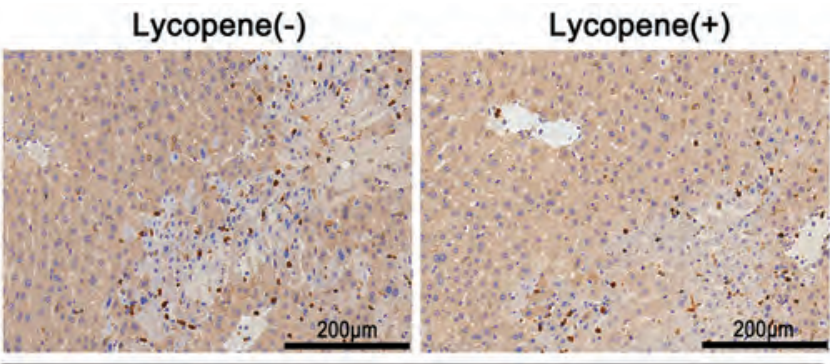

IR

Lycopene(-)

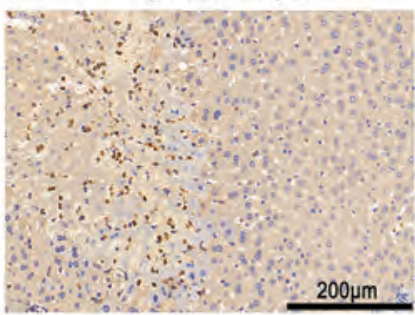

$200 \mu \mathrm{m}$

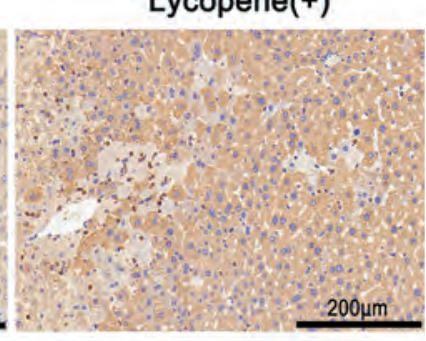

IR

E
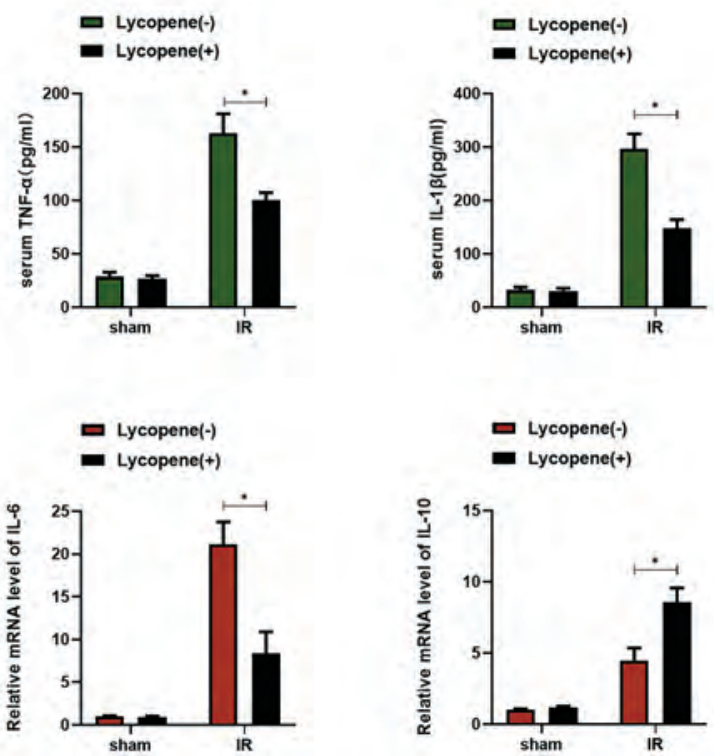

Figure 2 Lycopene attenuates the acute inflammatory responses in IR-stressed livers. (A,C) IHC staining of F4/80+ macrophages in liver sections (scale bars, $200 \mu \mathrm{m}$ ) and quantification of F4/80+ macrophages per high power field from different groups. (B,D) IHC staining of Ly6G+ neutrophils in liver sections (scale bars, $200 \mu \mathrm{m}$ ) and quantification of Ly6G+ neutrophils per high power field from different groups. (E) Levels of serum cytokines TNF- $\alpha$ and IL-6 measured by ELISA. (F) Quantitative RT-PCR analysis of relative mRNA levels of the cytokines TNF- $\alpha$, IL-1 $\beta$, IL-6, and IL-10. All data represent the mean $\pm \mathrm{SD}, \mathrm{n}=6$ mice/group, ${ }^{*} \mathrm{P}<0.05$. IR, ischemia reperfusion; IHC, immunohistochemical; TNF- $\alpha$, tumor necrosis factor- $\alpha$; IL-6, interleukin 6. 


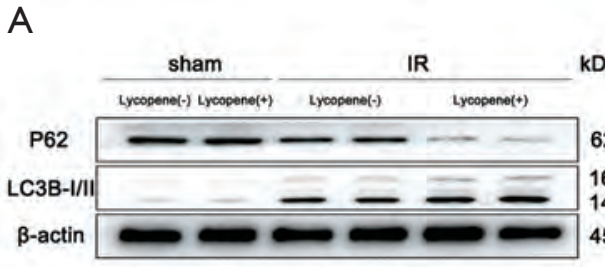

C

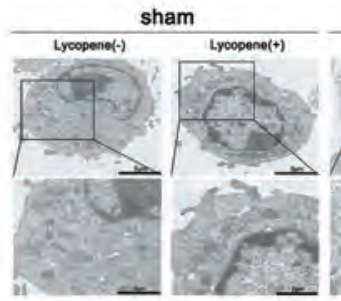

$\mathrm{F}$

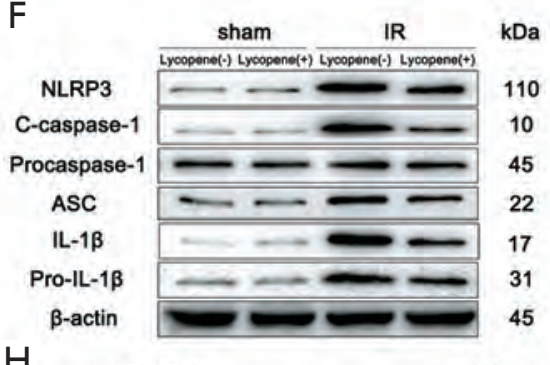

$\mathrm{H}$

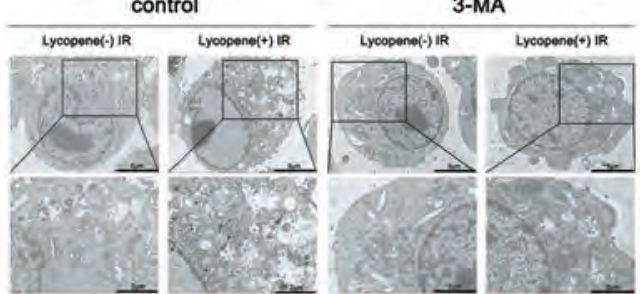

J

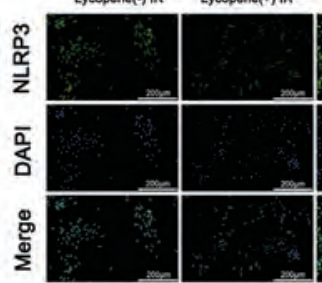

3-MA

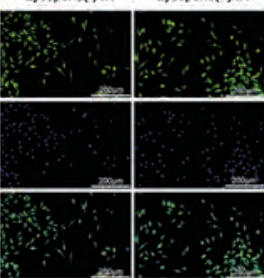

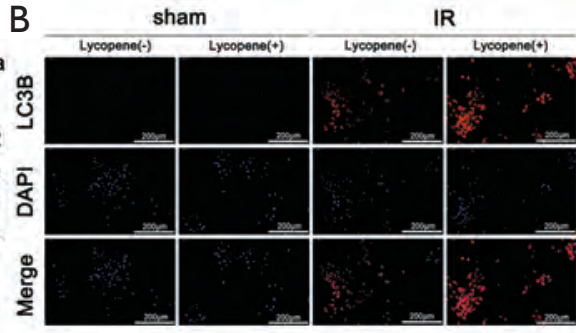

$\mathrm{D}$

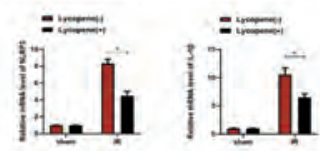

$E$
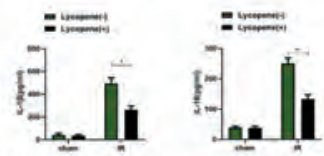

G
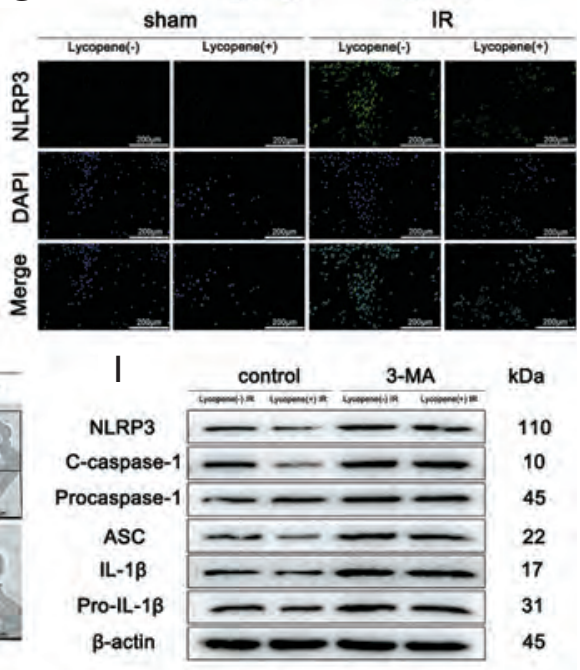

K

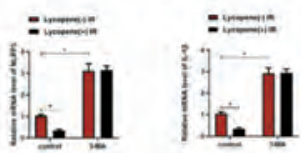

L

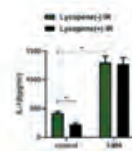

Figure 3 Lycopene inhibits NLRP3 inflammasome activation in Kupffer cells (KCs) through enhanced autophagy in IR-stressed livers. (A) Western blot analysis of the protein levels of $\mathrm{p} 62, \mathrm{LC} 3 \mathrm{~B}$, and $\beta$-actin in $\mathrm{KCs}$ isolated from different groups. (B) Immunofluorescence staining of LC3B in KCs isolated from different groups (scale bars, $200 \mu \mathrm{m}$ ). (C) Autophagic microstructures in KCs isolated from different groups detected by transmission electron microscopy (scale bars, 5 and $2 \mu \mathrm{m}$ ). (D) Quantitative RT-PCR analysis of relative mRNA levels of NLRP3 and IL- $1 \beta$ in KCs. (E) Levels of IL-1 $\beta$ and IL-18 in the culture supernatants of KCs isolated from different groups after $6 \mathrm{~h}$ of culture measured by ELISA. (F) Western blot analysis of the protein levels of NLRP3, cleaved caspase-1, procaspase-1, ASC, IL-1 $\beta$, proIL-1 $\beta$, and $\beta$-actin. (G) Immunofluorescence staining of NLRP3 in KCs isolated from different groups (scale bars, $200 \mu \mathrm{m})$. (H) Autophagic microstructures in KCs isolated from different groups detected by transmission electron microscopy (scale bars, 5 and $2 \mu \mathrm{m}$ ). (I) Western blot analysis of the protein levels of NLRP3, cleaved caspase-1, procaspase-1, ASC, IL-1 $\beta$, pro-IL-1 $\beta$, and $\beta$-actin. (J) Immunofluorescence staining of NLRP3 in KCs isolated from different groups (scale bars, $200 \mu \mathrm{m})$. (K) Quantitative RT-PCR analysis of relative mRNA levels of NLRP3 and IL-1 $\beta$ in KCs. (L) Levels of IL-1 $\beta$ and IL-18 in the culture supernatants of KCs isolated from different groups after $6 \mathrm{~h}$ of culture measured by ELISA. All data represent the mean $\pm \mathrm{SD},{ }^{*} \mathrm{P}<0.05$. 
stressed mice in the lycopene pretreatment group, compared to KCs isolated from IR-stressed mice in the control group (Figure 3G). Therefore, lycopene enhanced autophagic activity and suppressed NLRP3 inflammasome activation in KCs. To figure out whether lycopene inhibited NLRP3 inflammasome activation via enhancing autophagy in KCs isolated from IR-stressed mice, we pretreated mice from the 2 groups with 3-MA and inhibited autophagic activity. Our data showed that 3-MA abolished the enhancement of autophagic levels in KCs induced by lycopene (Figure $3 H$ ). This resulted in enhanced NLRP3 inflammasome activation in KCs, which was determined based on the markedly elevated protein levels of NLRP3, cleaved caspase-1, ASC, IL-1 $\beta$, and pro-IL-1 $\beta$ (Figure 3I), as well as increased NLRP3 fluorescence intensity (Figure 37). Furthermore, the relative mRNA expression levels of NLRP3 and IL-1 $\beta$ were significantly elevated in KCs after pretreatment with 3 -MA in the 2 groups (Figure $3 K$ ). In line with the above data, 3-MA pretreatment abolished the decreased levels of IL- $1 \beta$ and IL-18 in the supernatants of KCs isolated from IR-stressed mice (Figure 3L).

Pretreatment with 3-MA also abolished the protective effects of lycopene in IR-stressed mice, as evidenced by the elevated serum ALT and AST levels (Figure 4A), severe sinusoidal congestion, edema, vacuolization, or necrosis (Figure 4B), a higher Suzuki score (Figure 4C), and increased MPO activity (Figure 4D). Also, the number of TUNELpositive hepatocytes and caspase- 3 relative activity both significantly increased in mice pretreated with lycopene or olive oil after pretreatment with 3-MA (Figure 4E,F,G). Consistent with these results, the western blotting results showed that 3-MA pretreatment inhibited the expression of Bcl-2 and Bcl-xL (Figure 4H).

\section{Lycopene inbibits NLRP3 inflammasome activation by activating Nrf2-mediated KC autophagy during hepatic IR injury}

It was reported that lycopene could serve as an activator of the Nrf2/HO-1 pathway $(42,43)$. Many studies have demonstrated that Nrf2 regulates autophagy activity $(44,45)$. Therefore, we set out to assess whether the enhancement of autophagic levels in KCs induced by lycopene depends on the expression of Nrf2. The western blotting results showed that the expression of nuclear Nrf2 and total HO-1 was increased, and the expression cytoplasmic Nrf2 was decreased in KCs isolated from mice pretreated with lycopene (Figure $5 A$ ). To further determine the function of $\mathrm{Nrf} 2$ in regulating the autophagic levels of KCs, mannose-conjugated polymers were utilized to deliver Nrf2 siRNA (Nrf2-siRNA) or scrambled control siRNA (SCR-siRNA) in vivo. Mannose-mediated siRNA express a mannose-specific membrane receptor, which can be particularly delivered to macrophages/KCs (46). As shown in Figure 5B, the protein levels of $\mathrm{Nrf2}$ in both the nucleus and cytoplasm were efficiently knocked down by Nrf2siRNA, and correspondingly, the protein levels of HO-1 and LC3B were significantly decreased, and the protein levels of p62 were markedly increased. The immunofluorescence staining results showed lower LC3B expression in the Nrf2siRNA IR groups pretreated with lycopene, indicating that the enhanced autophagy in KCs induced by lycopene was blocked (Figure 5C). To further analyze the autophagic levels, transmission electron microscopy was applied to detect autophagosomes in KCs isolated from IR-stressed livers. The results demonstrated that Nrf2-siRNA abolished the enhanced autophagy induced by lycopene, based on reduced autophagosomes in the Nrf2-siRNA IR groups (Figure 5D). These data confirmed that lycopene enhanced KC autophagy activity via increasing the expression of $\mathrm{Nrf2}$.

Meanwhile, the NLRP3 inflammasome was activated in $\mathrm{KCs}$ isolated from the lycopene pretreatment group when Nrf2 was knocked down, evidenced by the increased protein levels of NLRP3, cleaved caspase-1, ASC, IL-1 $\beta$, and proIL-1 $\beta$ (Figure $5 E$ ). The immunofluorescence staining results displayed that NLRP3 expression was elevated in the Nrf2siRNA IR groups (Figure 5F). Consistent with the above data, the mRNA levels of NLRP3 and IL- $1 \beta$ and the levels of secreted IL- $1 \beta$ and IL- 18 were higher in KCs isolated from the Nrf2-siRNA IR groups (Figure $5 G, H$ ).

Furthermore, Nrf2 knockdown abolished lycopene's protective effect in mice during hepatic IR injury (Figure $6 A, B, C, D, E, F, G, H)$. These results demonstrated that lycopene suppressed NLRP3 inflammasome activation via increasing the expression of $\mathrm{Nrf2}$ and enhancing $\mathrm{KC}$ autophagy during hepatic IR injury.

\section{HO-1 was indispensable for Nrf2-mediated KC autophagy enhanced by lycopene during bepatic IR injury}

It was reported that $\mathrm{HO}-1$, as a downstream target gene of Nrf2, played an essential role in regulating autophagic activity and acute hepatic injury $(47,48)$. Therefore, we wanted to determine whether the autophagic activity of KCs enhanced by lycopene depends on the expression of HO-1. Mannose-conjugated polymers were employed to deliver 
A

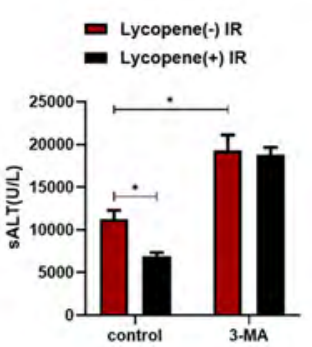

C

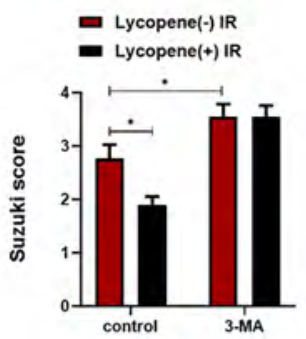

E

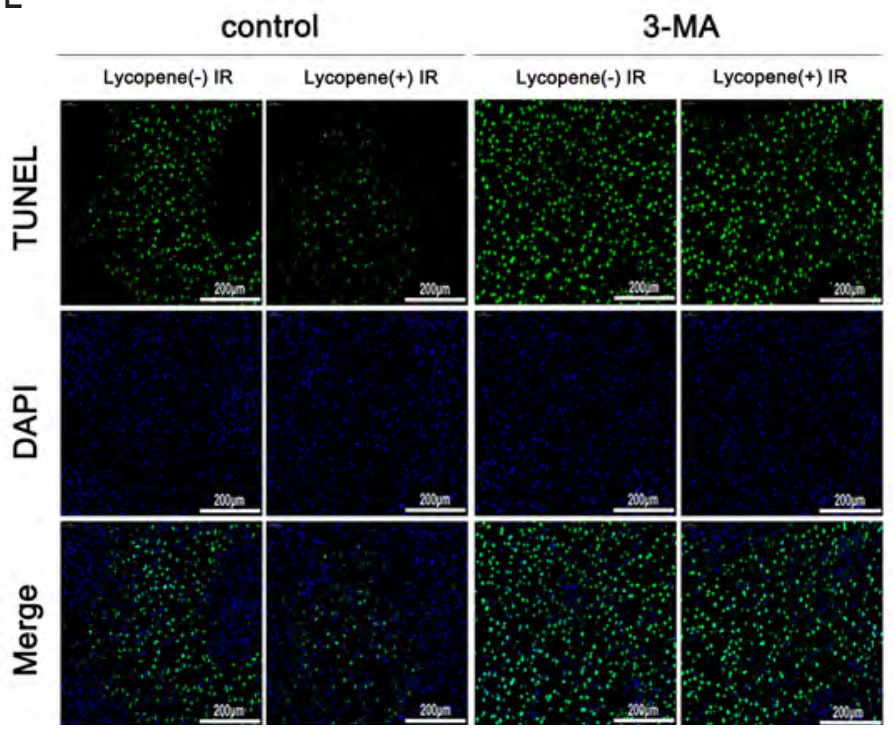

B

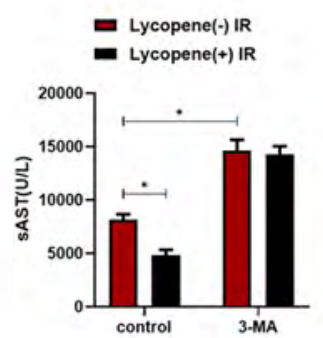

\section{홍}

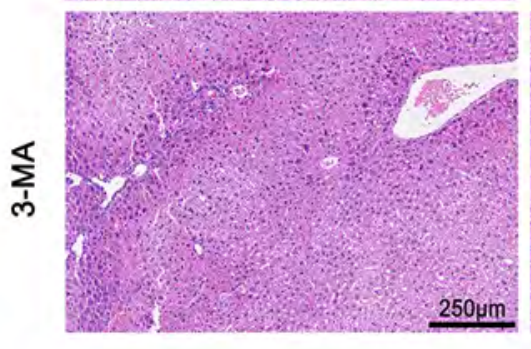

F

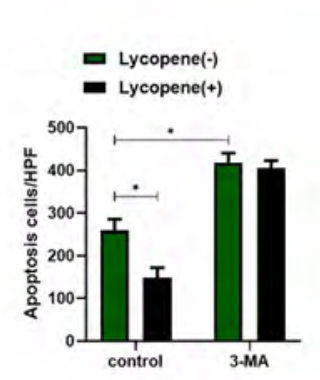

I

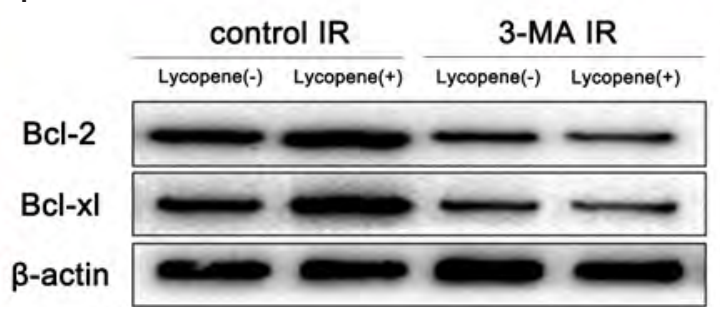

Lycopene(+) IR
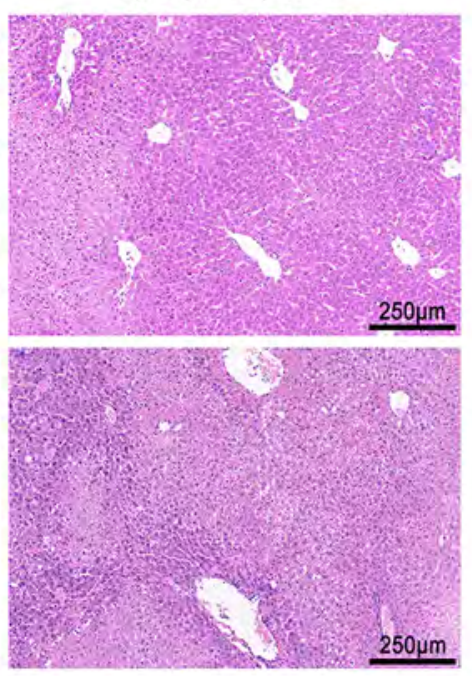

G

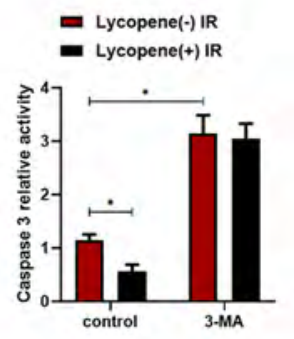

$\mathrm{kDa}$

26

30

45

Figure 4 Pretreatment with 3-MA exacerbates the IR-induced acute liver injury in mice pretreated with lycopene. (A) Serum ALT and AST in mice with IR injury in different groups ( $\mathrm{n}=6$ mice/group). (B) Representative hematoxylin and eosin (H\&E) staining of liver tissue sections for different groups (scale bars, $250 \mu \mathrm{m})$. (C,D) The average Suzuki scores and liver myeloperoxidase (MPO) activities for different groups. (E,F) TUNEL staining of liver sections (scale bars, $200 \mu \mathrm{m}$ ) and the relative ratios of TUNEL-positive cells for different groups. (G) Relative caspase-3 activity was evaluated from total lysates of IR lobes. (H) Western blot analysis of the protein levels of Bcl-2, Bcl-xL, and $\beta$-actin in different groups. All data represent the mean $\pm \mathrm{SD},{ }^{*} \mathrm{P}<0.05$. IR, ischemia reperfusion; ALT, alanine aminotransferase; AST, aspartate aminotransferases.

HO-1 siRNA (HO-1-siRNA) or scrambled control siRNA (SCR-siRNA) in vivo. As shown in Figure $7 A$, the protein levels of LC3B were downregulated, and the protein levels of p62 were upregulated when HO-1-siRNA efficiently knocked down HO-1. The knockdown of HO-1 in KCs suppressed the autophagic activity enhanced by lycopene in IR-stressed mice, evidenced by the lower expression of LC3B and the smaller number of autophagosomes (Figure $7 B, C$ ). Meanwhile, the knockdown of HO-1 in KCs restored and elevated NLRP3 inflammasome activation 

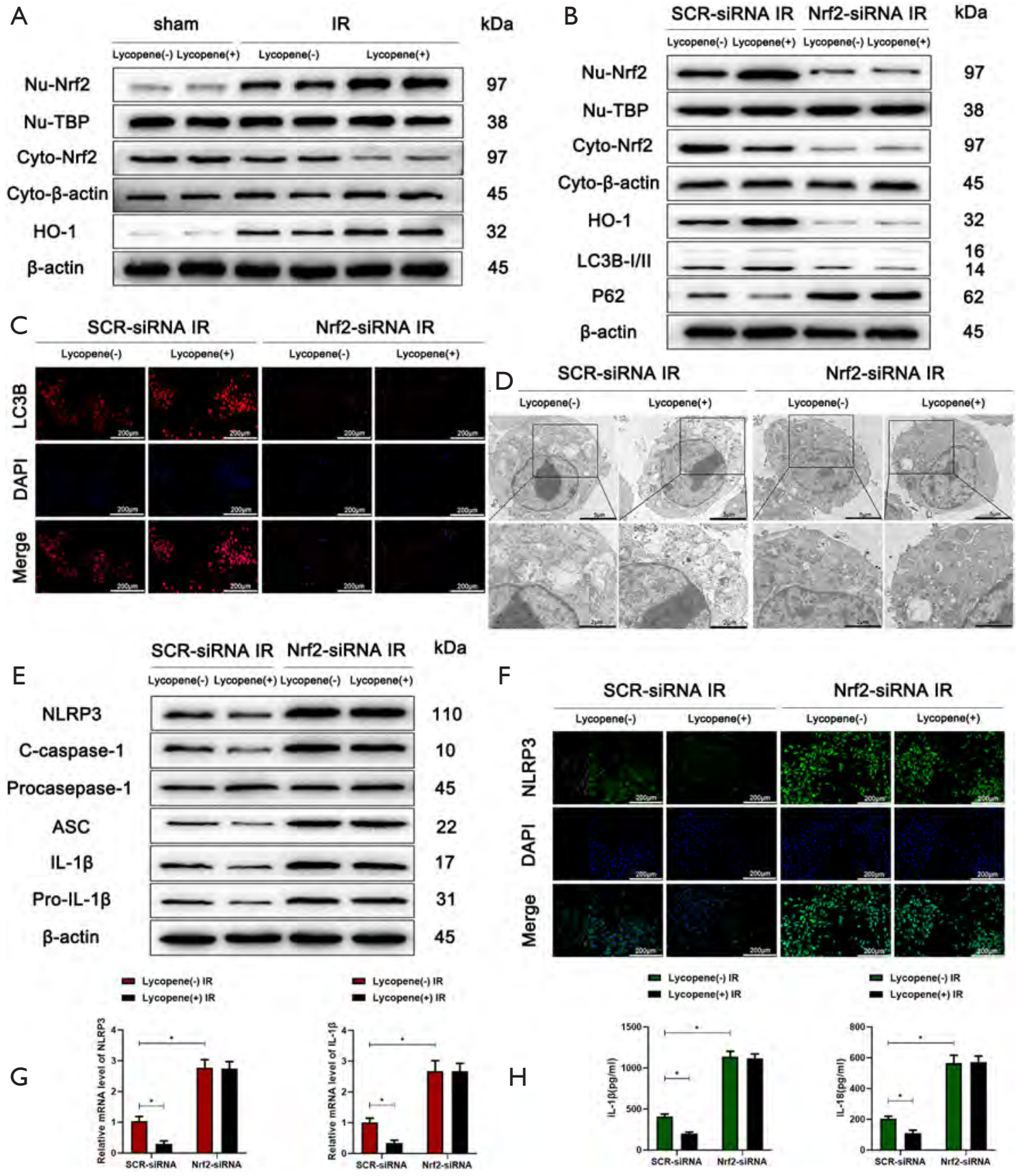

Figure 5 Lycopene activates Nrf2-mediated Kupffer cell (KC) autophagy in hepatic IR injury. (A) Western blot analysis of the protein levels of HO-1 and nuclear and cytoplasmic Nrf2 in KCs isolated from mice who underwent sham surgery or IR injury. (B) Western blot analysis of the protein levels of intracellular HO-1, LC3B, p62, and nuclear and cytoplasmic Nrf2 in KCs isolated from mice who underwent IR injury in different groups ( $\mathrm{n}=5$ mice/group). (C) Immunofluorescence staining of LC3B in KCs isolated from different groups (scale bars, $200 \mu \mathrm{m}$ ). (D) Autophagic microstructures in KCs isolated from different groups detected by transmission electron microscopy (scale bars, 5 and $2 \mu \mathrm{m}$ ). (E) Western blot analysis of the protein levels of NLRP3, cleaved caspase-1, procaspase-1, ASC, IL-1 $\beta$, pro-IL-1 $\beta$, and $\beta$-actin. (F) Immunofluorescence staining of NLRP3 in KCs isolated from different groups (scale bars, $200 \mu \mathrm{m})$. (G) Quantitative RT-PCR analysis of the relative mRNA levels of NLRP3 and IL-1 in KCs isolated from different groups. (H) Levels of IL-1 $\beta$ and IL-18 in the culture supernatants of KCs isolated from different groups after $6 \mathrm{~h}$ of culture measured by ELISA. All data represent the mean $\pm \mathrm{SD},{ }^{*} \mathrm{P}<0.05$. 
A

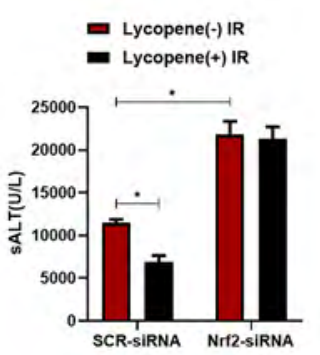

C

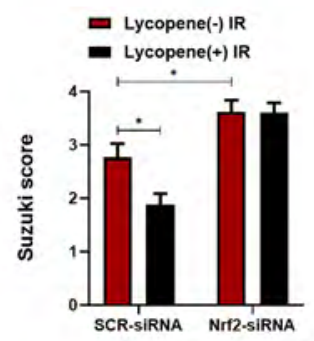

E

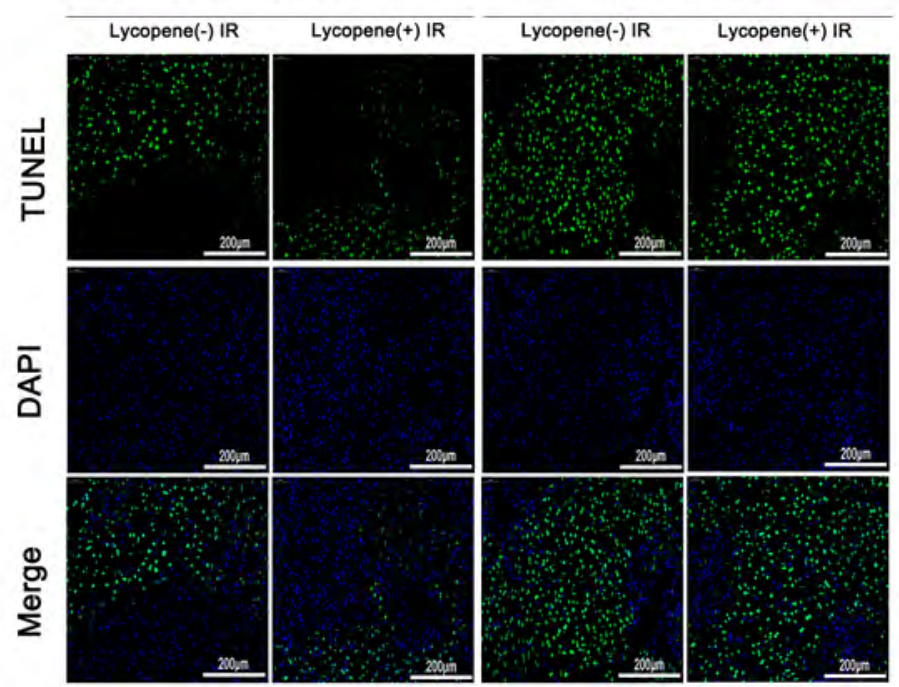

B

Lycopene(-) IR

- Lycopene(+) IR

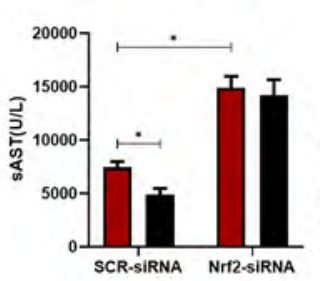

D

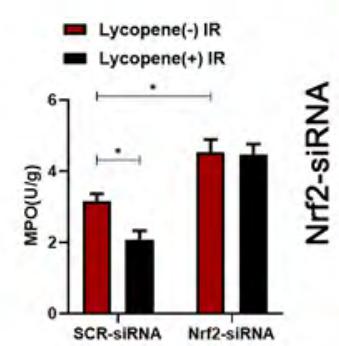

Lycopene(-) IR
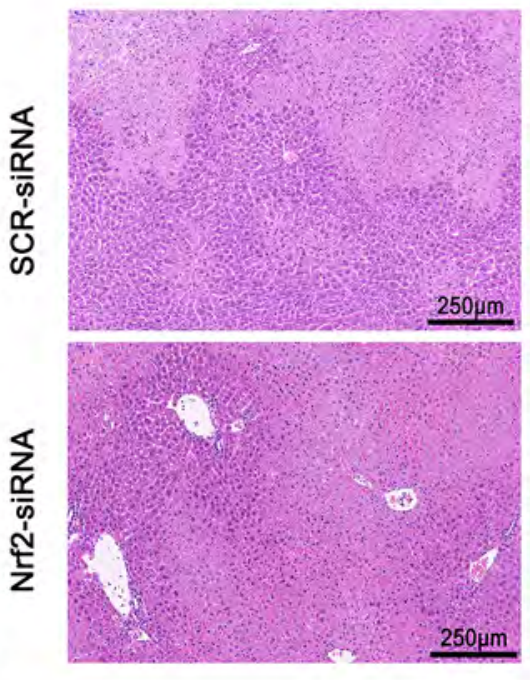

F
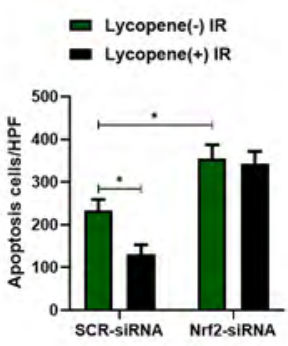

Lycopene(+) IR
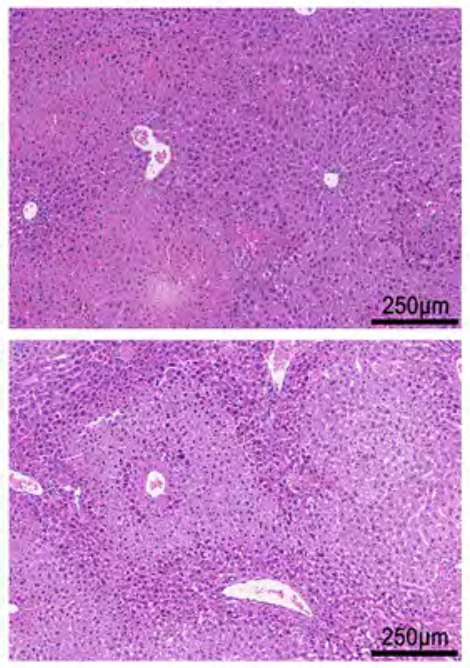

$\mathrm{H}$

G

$$
\begin{aligned}
& \text { Lycopene(-) IR } \\
& \text { Lycopene(+) IR }
\end{aligned}
$$

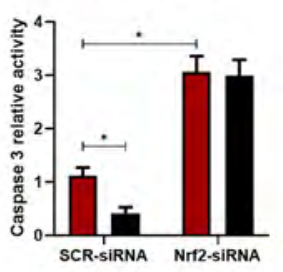

\section{SCR-SiRNA IR}

\section{Nrf2-siRNA IR}

$\mathrm{kDa}$

$\mathrm{Bcl}-2$

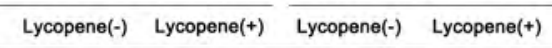

$\mathrm{Bcl}-\mathrm{xl}$

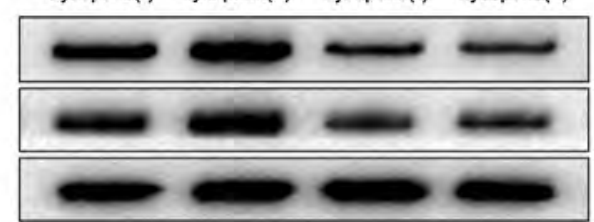

26

30

$\beta$-actin

Figure 6 Nrf2 knockdown in Kupffer cells (KCs) exacerbates IR-induced acute liver injury. (A) Serum ALT and AST in mice who underwent IR injury in different groups ( $\mathrm{n}=5$ mice/group). (B) Representative haematoxylin and eosin (HE) staining of liver tissue sections from different groups (scale bars, $250 \mu \mathrm{m}$ ). (C,D) The average Suzuki scores and liver myeloperoxidase (MPO) activities of different groups. (E,F) TUNEL staining of liver sections (scale bars, $200 \mu \mathrm{m}$ ) and the relative ratios of TUNEL-positive cells in different groups. (G) Relative caspase-3 activity was evaluated from total lysates of IR lobes from different groups. $(\mathrm{H})$ Western blot analysis of the protein levels of Bcl-2, $\mathrm{Bcl}-\mathrm{xL}$, and $\beta$-actin in different groups. All data represent the mean $\pm \mathrm{SD},{ }^{*} \mathrm{P}<0.05$. IR, ischemia reperfusion; ALT, alanine aminotransferase; AST, aspartate aminotransferases. 
A

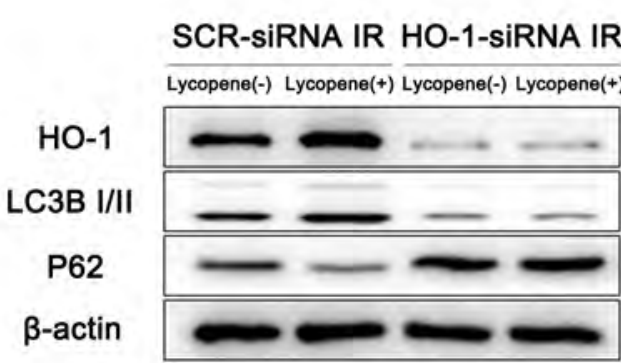

C

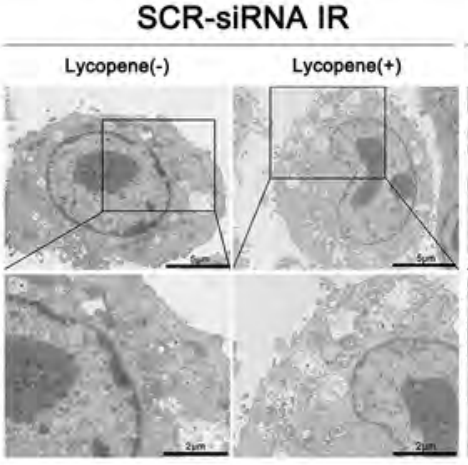

D

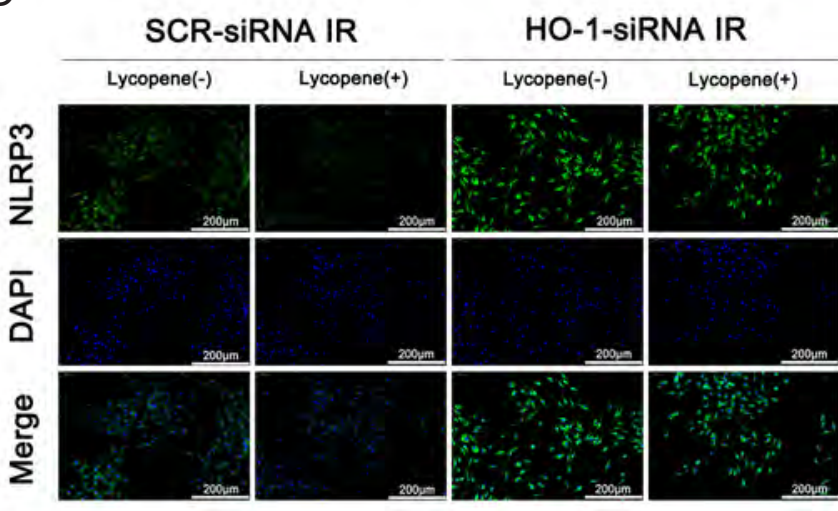

$\mathrm{F}$
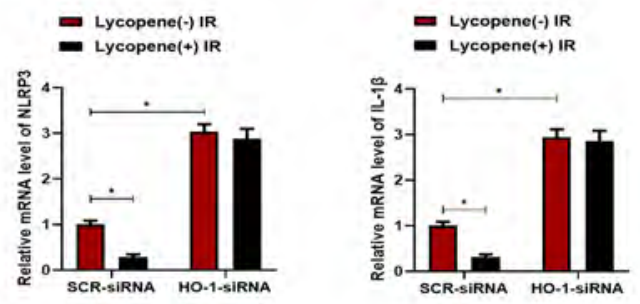

G

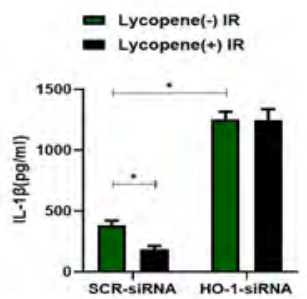

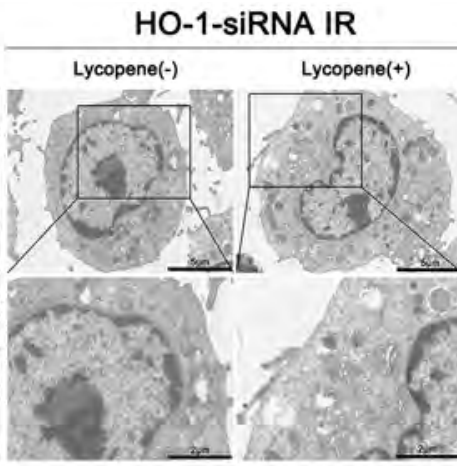

B

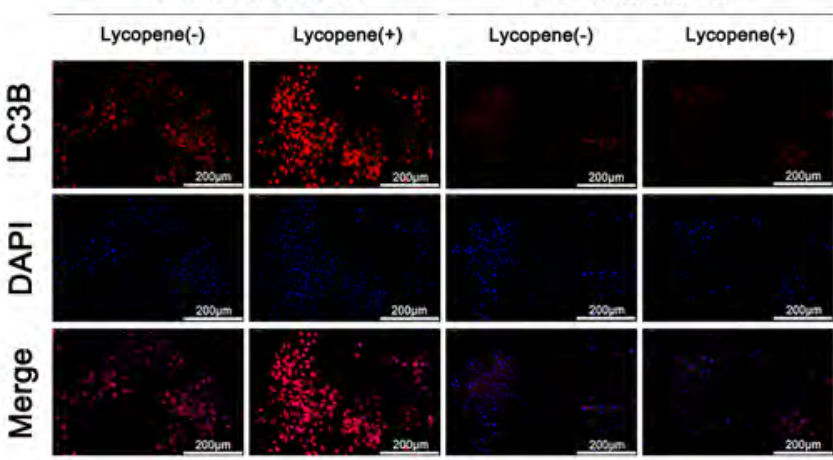

$E$

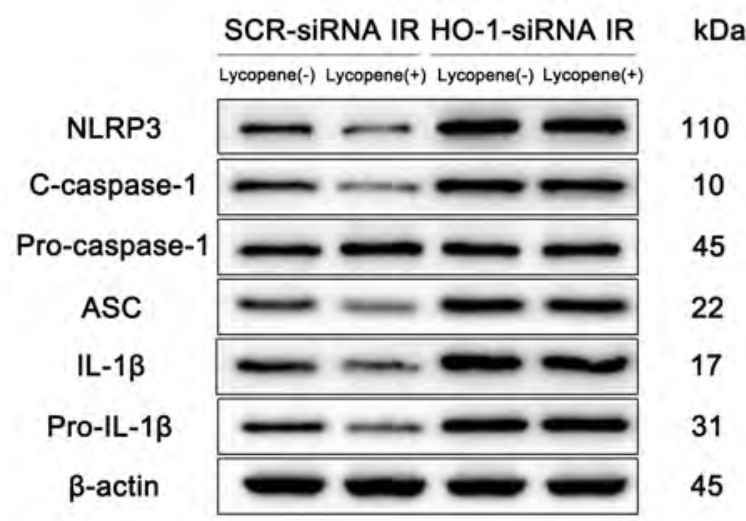

$\mathrm{kDa}$

10

45

45

Figure 7 HO-1 knockdown inhibits Nrf2-mediated autophagy and promotes the expression of the NLRP3 inflammasome in Kupffer cells (KCs). (A) Western blot analysis of the protein levels of HO-1, LC3B, p62, and $\beta$-actin in KCs isolated from mice who underwent IR injury in different groups ( $\mathrm{n}=5$ mice/group). (B) Immunofluorescence staining of LC3B in KCs isolated from different groups (scale bars, $200 \mu \mathrm{m}$ ). (C) Autophagic microstructures in KCs isolated from different groups detected by transmission electron microscopy (scale bars, 5 and $2 \mu \mathrm{m}$ ). (D) Immunofluorescence staining of NLRP3 in KCs isolated from different groups (scale bars, $200 \mu \mathrm{m}$ ). (E) Western blot analysis of the protein levels of NLRP3, cleaved caspase-1, procaspase-1, ASC, IL-1 $\beta$, pro-IL-1 $\beta$, and $\beta$-actin. (F) Quantitative RT-PCR analysis of the relative mRNA levels of NLRP3 and IL-1 $\beta$ in KCs isolated from different groups. (G) Levels of IL-1 $\beta$ and IL-18 in the culture supernatants of KCs isolated from different groups after $6 \mathrm{~h}$ of culture measured by ELISA. All data represent the mean $\pm \mathrm{SD},{ }^{*} \mathrm{P}<0.05$. 
in mice pretreated with lycopene during hepatic IR injury (Figure 7D). Consistently, the protein levels of NLRP3, cleaved caspase- 1 , ASC, IL- $1 \beta$, and pro-IL-1 $\beta$ were upregulated (Figure $7 E$ ). The mRNA levels of NLRP3 and IL- $1 \beta$ and the levels of secreted IL- $1 \beta$ and IL- 18 were increased (Figure $7 F, G$ ). Furthermore, for mice treated with HO-1-siRNA, lycopene's protective effects were abolished during hepatic IR injury (Figure $8 A, B, C, D, E, F, G, H$ ). These results indicated that lycopene-mediated protection against hepatic IR injury depended on the expression of HO-1. In conclusion, our results identified that lycopene inhibited NLRP3 inflammasome activation via activating the Nrf2/ HO-1 pathway and enhancing $\mathrm{KC}$ autophagy during hepatic IR injury.

\section{Discussion}

The occurrence of severe hepatic IR injury can prolong hospital stays and increase treatment costs (49). To specifically attenuate hepatic IR injury, much effort has been directed towards researching the mechanisms (1). For example, it was reported that small-molecule inhibitors of cyclophilins protected mice from hepatic IR injury via blocking the opening of the mitochondrial permeability transition pore (50). Another study demonstrated that hepatocyte cyclooxygenase-2 exerted a protective function against hepatic IR injury in mice (51). Meanwhile, our previous study showed that the plasma membrane-bound G protein-coupled bile acid receptor (TGR5) reduced hepatic IR injury by suppressing TLR4-NF- $\kappa$ B pathway activation (52). During our present study, we demonstrated that lycopene could also alleviate hepatic IR injury. Phenotypically, lycopene reduces hepatocellular damage, necrosis, and apoptosis.

It has been reported that lycopene plays a crucial role in protecting organs from IR injury. For example, lycopene protected cardiomyocytes from IR injury via inhibiting mtDNA oxidative damage (34). Another study suggested that lycopene reduced cerebral IR injury via regulating iron metabolism (33). Although a study indicated that lycopene had a protective effect against hepatic IR injury in rats (35), the underlying mechanisms have not been elucidated.

$\mathrm{KCs}$, as one kind of NPC from the liver, play a vital role in maintaining hepatic immune homeostasis (53). It has been reported that KCs are involved in acute liver injuries, such as drug-induced liver injury and hepatic IR injury. For example, a study showed that MyD88-dependent activation of IL$1 \alpha$ in KCs promoted sterile inflammation in APAP-induced acute hepatic injury (54). Another study demonstrated that roquin-1 affected the polarization of KCs through regulating AMPK $\alpha$ activity following hepatic IR injury (55). Meanwhile, it has been reported that lycopene attenuated obesity-induced inflammation and insulin resistance via regulating macrophage polarization (56). However, the effects of lycopene on KCs in IR-stressed livers remains unknown. In our present study, lycopene reduced the accumulation of inflammatory cells and attenuated acute inflammatory immune responses.

The NLRP3 inflammasome plays a crucial role in various pathological processes, including IR injury (57). For example, NLRP3 inflammasome activation induced by mitochondrial dysfunction aggravated cerebral IR injury (58). One study showed that the inhibition of the NLRP3 inflammasome could alleviate myocardial IR injury in mice (59). A recent study showed that the NLRP3 inflammasome was inhibited by Jagged1-mediated myeloid Notch1 signaling through promoting heat shock transcription factor 1 (HSF1) and Snail activation, which led to reduced hepatic IR injury (60). Another important investigation demonstrated that PINK1mediated mitophagy attenuated hepatic IR injury by inhibiting NLRP3 inflammasome activation (61). Although several NLRP3 inhibitors have been found and synthesized, such as parthenolide, VX-740 (pralnacasan) and its analog VX-765, bay 11-7082 (a phenyl vinyl sulfone), and ß-hydroxybutyrate, there are still no applications for hepatic IR injury. These drugs cannot be used in the liver due to significant hepatotoxicity, which necessitates the discovery of better inhibitors. However, whether lycopene affects the NLRP3 inflammasome in KCs in hepatic IR injury remains unknown. Our present study showed that lycopene could inhibit the activation of the NLRP3 inflammasome in KCs in hepatic IR injury.

It is well known that autophagy is widely involved in the liver's physiological and pathological state (62). A study showed that the enhancement of autophagy in macrophages reduced acute hepatic toxic injury induced by $\mathrm{D}$-galactosamine/lipopolysaccharide through downregulating IL-1 $\beta$ expression in mice (63). Another study indicated that $\mathrm{T}$ cell $\mathrm{Ig}$ mucin domain protein 4 (Tim-4) inhibited NLRP3 inflammasome activation via activating liver kinase B1/AMPK $\alpha$-mediated autophagy in macrophages in nonalcoholic fatty liver disease (64). Our previous study demonstrated that spermine attenuated acute TAA-induced hepatic injury through suppressing KC pro-inflammatory responses and enhancing ATG5dependent autophagic levels (65). Meanwhile, it was reported that ezetimibe suppressed NLRP3 inflammasome 
A

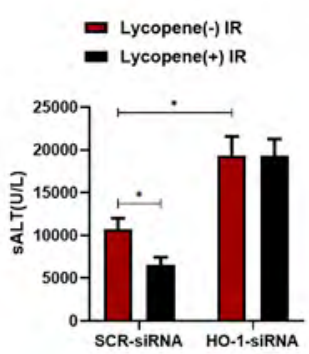

C

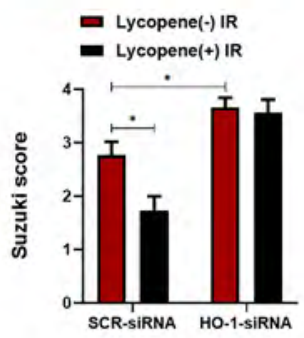

E

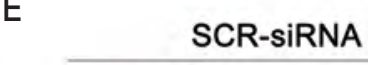

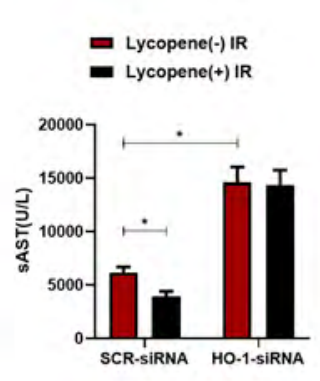

D $\quad$ Lycopene(-) IR

- Lycopene(+) IR

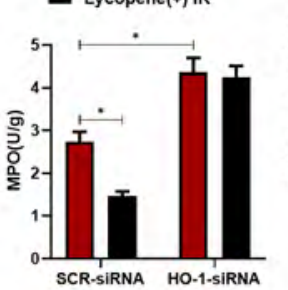

B

$$
\begin{aligned}
& \overleftarrow{z} \\
& \frac{x}{w} \\
& \frac{1}{0} \\
& 0 \\
& 0
\end{aligned}
$$

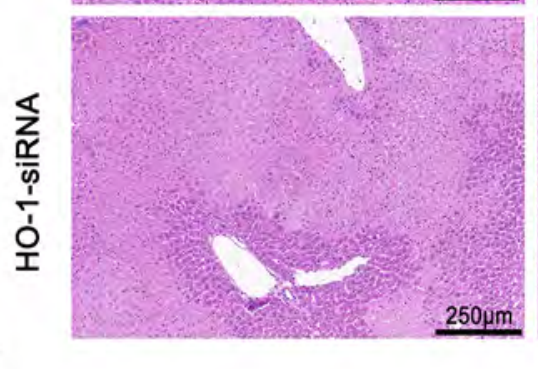

$\mathrm{F}$

Lycopene(-) IR
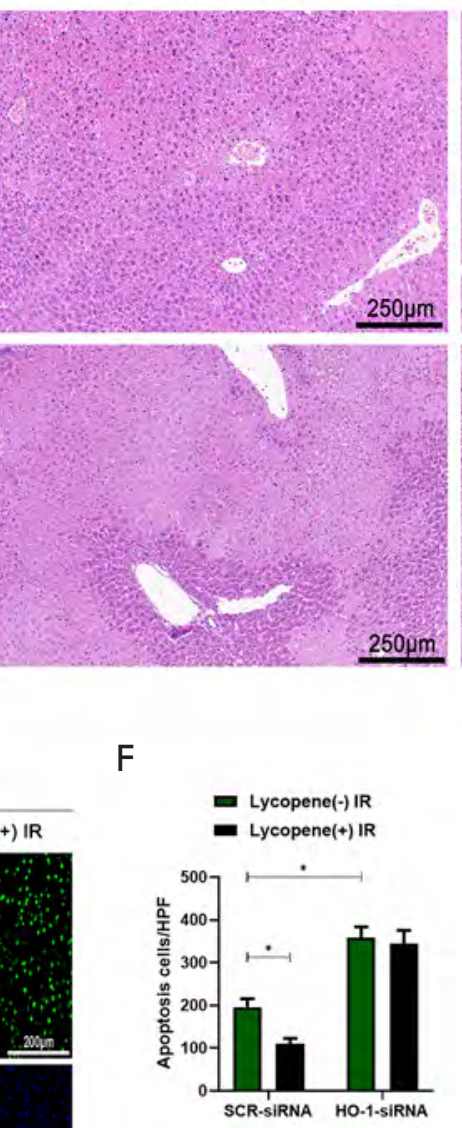

$\mathrm{H}$

Bcl-2

Bcl-xl

$\beta$-actin

\section{B-actin}

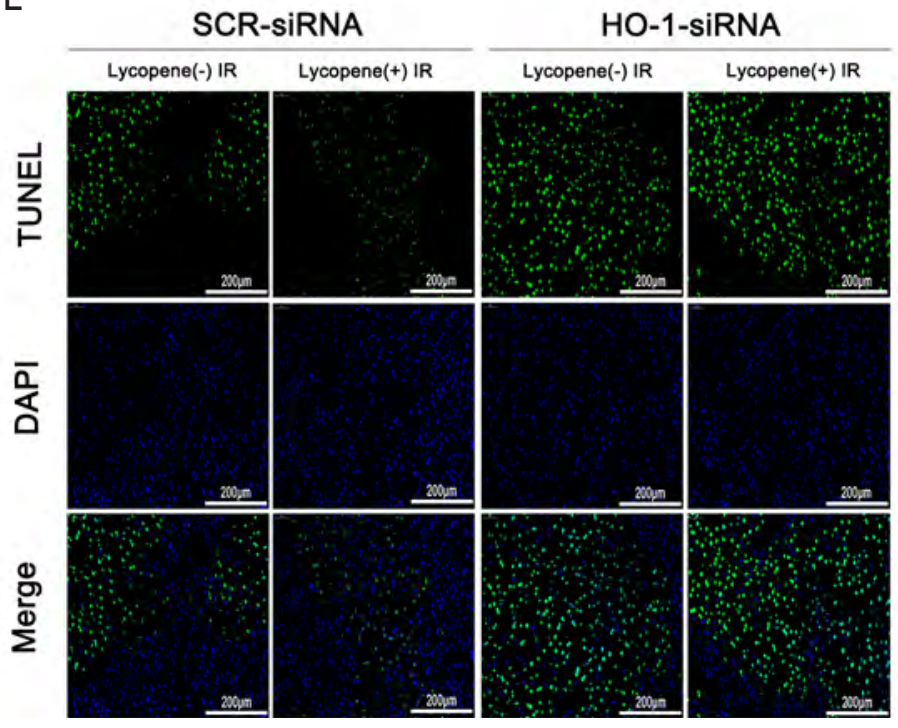

G

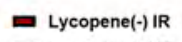

- Lycopene(+) IR

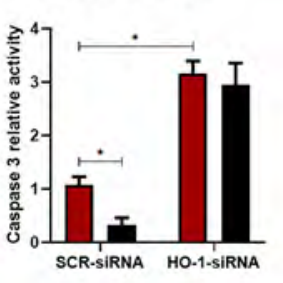

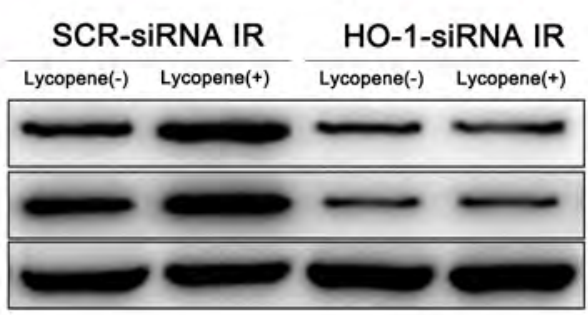

$\mathrm{kDa}$ 26

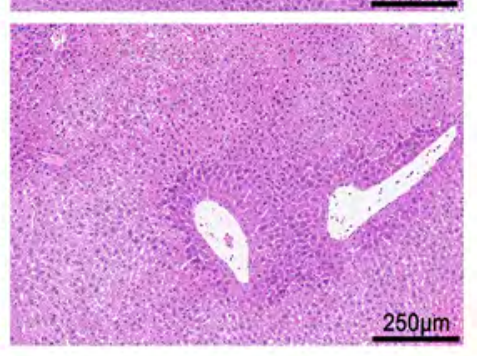

Lycopene(+) IR

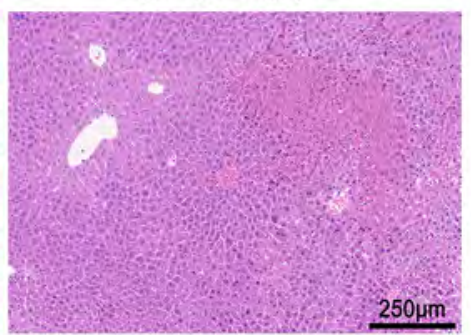

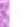

Figure 8 HO-1 knockdown in Kupffer cells (KCs) exacerbates IR-induced acute liver injury. (A) Serum ALT and AST in mice who
underwent IR injury in different groups ( $\mathrm{n}=5$ mice/group). (B) Representative hematoxylin and eosin (H\&E) staining of liver tissue sections from different groups (scale bars, $250 \mu \mathrm{m}$ ). (C,D) The average Suzuki scores and liver myeloperoxidase (MPO) activities in different groups. (E,F) TUNEL staining of liver sections (scale bars, $200 \mu \mathrm{m}$ ) and the relative ratios of TUNEL-positive cells in different groups. (G) Relative caspase-3 activity was evaluated from total lysates of IR lobes from different groups. (H) Western blot analysis of the protein levels of Bcl-2, Bcl-xL, and $\beta$-actin for different groups. All data represent the mean $\pm \mathrm{SD},{ }^{*} \mathrm{P}<0.05$. IR, ischemia reperfusion; ALT, alanine aminotransferase; AST, aspartate aminotransferases.

activation in macrophages via modulating autophagic levels in nonalcoholic steatohepatitis (66). Our data showed that the autophagy enhanced by lycopene suppressed NLRP3 inflammasome activation in KCs. The autophagy inhibitor
3-MA was able to abolish lycopene's protective effects. The autophagic activity enhanced by lycopene in KCs depended on the Nrf2/HO-1 pathway. When Nrf2-siRNA knocked down Nrf2 in KCs, lycopene failed to enhance the 
autophagic activity, which resulted in NLRP3 inflammasome activation and aggravated hepatic IR injury in mice.

Similarly, HO-1 knocked down by HO-1-siRNA in KCs decreased autophagic activity and activated the NLRP3 inflammasome. Therefore, the Nrf2/HO-1 pathway activated by lycopene suppressed NLRP3 inflammasome activation and alleviated hepatic IR injury via enhancing autophagy. Although the definite mechanisms of autophagy induction by $\mathrm{HO}-1$ remain unclear, we speculate that carbon monoxide, a product of HO-1 activity, may serve as a key factor to stimulate autophagy $(67,68)$.

In conclusion, our present study demonstrated that lycopene enhanced Nrf2/HO-1-mediated autophagic activity, which resulted in reduced NLRP3 inflammasome activation in KCs and alleviated hepatic IR injury. Our findings suggest that lycopene may possess potential therapeutic value in hepatic IR injury.

\section{Acknowledgments}

Funding: This study was supported by the National Natural Science Foundation of China (grant numbers 81971495), Research Unit of Liver Transplantation and Transplant Immunology, Chinese Academy of Medical Sciences (grant number 2019-I2M-5-035), and State Key Laboratory of Reproductive Medicine, Nanjing Medical University (SKLRM-K202001).

\section{Footnote}

Reporting Checklist: The authors have completed the ARRIVE reporting checklist. Available at http://dx.doi. org/10.21037/atm-20-7084

Data Sharing Statement: Available at http://dx.doi. org/10.21037/atm-20-7084

Conflicts of Interest: All authors have completed the ICMJE uniform disclosure form (available at http://dx.doi. org/10.21037/atm-20-7084). LL serves as an unpaid Associate Editor-in-Chief of Annals of Translational Medicine from Jun 2019 to May 2024. The other authors have no conflicts of interest to declare.

Ethical Statement: The authors are accountable for all aspects of the work in ensuring that questions related to the accuracy or integrity of any part of the work are appropriately investigated and resolved. The animal experiments in this study were approved by the Ethics Committee of Nanjing Medical University (Approval No.: IACUC-1912015), in compliance with the Nanjing Medical University guidelines for the care and use of animals.

Open Access Statement: This is an Open Access article distributed in accordance with the Creative Commons Attribution-NonCommercial-NoDerivs 4.0 International License (CC BY-NC-ND 4.0), which permits the noncommercial replication and distribution of the article with the strict proviso that no changes or edits are made and the original work is properly cited (including links to both the formal publication through the relevant DOI and the license). See: https://creativecommons.org/licenses/by-nc-nd/4.0/.

\section{References}

1. Zhai Y, Petrowsky H, Hong JC, et al. Ischaemiareperfusion injury in liver transplantation--from bench to bedside. Nat Rev Gastroenterol Hepatol 2013;10:79-89.

2. Marshall K, Jin J, Atkinson C, et al. Natural immunoglobulin $M$ initiates an inflammatory response important for both hepatic ischemia reperfusion injury and regeneration in mice. Hepatology 2018;67:721-35.

3. Vollmar B, Menger MD. The hepatic microcirculation: mechanistic contributions and therapeutic targets in liver injury and repair. Physiol Rev 2009;89:1269-339.

4. Zhou H, Wang H, Ni M, et al. Glycogen synthase kinase $3 \beta$ promotes liver innate immune activation by restraining AMP-activated protein kinase activation. J Hepatol 2018;69:99-109.

5. Ju C, Tacke F. Hepatic macrophages in homeostasis and liver diseases: from pathogenesis to novel therapeutic strategies. Cell Mol Immunol 2016;13:316-27.

6. Abu-Amara M, Yang SY, Tapuria N, et al. Liver ischemia/ reperfusion injury: processes in inflammatory networks--a review. Liver Transpl 2010;16:1016-32.

7. Kim HY, Kim SJ, Lee SM. Activation of NLRP3 and AIM2 inflammasomes in Kupffer cells in hepatic ischemia/ reperfusion. FEBS J 2015;282:259-70.

8. Huang H, Chen HW, Evankovich J, et al. Histones activate the NLRP3 inflammasome in Kupffer cells during sterile inflammatory liver injury. J Immunol 2013;191:2665-79.

9. Song N, Li T. Regulation of NLRP3 Inflammasome by Phosphorylation. Front Immunol 2018;9:2305.

10. Inoue $\mathrm{Y}$, Shirasuna K, Kimura H, et al. NLRP3 regulates neutrophil functions and contributes to hepatic ischemiareperfusion injury independently of inflammasomes. J 
Immunol 2014;192:4342-51.

11. Li C, Jin Y, Wei S, et al. Hippo Signaling Controls NLR Family Pyrin Domain Containing 3 Activation and Governs Immunoregulation of Mesenchymal Stem Cells in Mouse Liver Injury. Hepatology 2019;70:1714-31.

12. Yu L, Chen Y, Tooze SA. Autophagy pathway: Cellular and molecular mechanisms. Autophagy 2018;14:207-15.

13. Xue F, Hu L, Ge R, et al. Autophagy-deficiency in hepatic progenitor cells leads to the defects of stemness and enhances susceptibility to neoplastic transformation. Cancer Lett 2016;371:38-47.

14. Lin Y, Wu C, Wang X, et al. Glucosamine promotes hepatitis $\mathrm{B}$ virus replication through its dual effects in suppressing autophagic degradation and inhibiting MTORC1 signaling. Autophagy 2020;16:548-61.

15. Jiang T, Zhan F, Rao Z, et al. Combined ischemic and rapamycin preconditioning alleviated liver ischemia and reperfusion injury by restoring autophagy in aged mice. Int Immunopharmacol 2019;74:105711.

16. Chen X, Chan H, Zhang L, et al. The phytochemical polydatin ameliorates non-alcoholic steatohepatitis by restoring lysosomal function and autophagic flux. J Cell Mol Med 2019;23:4290-300.

17. Wang $\mathrm{Y}$, Meng $\mathrm{C}$, Zhang J, et al. Inhibition of GSK-3 $\beta$ alleviates cerebral ischemia/reperfusion injury in rats by suppressing NLRP3 inflammasome activation through autophagy. Int Immunopharmacol 2019;68:234-41.

18. Zhang D, He Y, Ye X, et al. Activation of autophagy inhibits nucleotide-binding oligomerization domain-like receptor protein 3 inflammasome activation and attenuates myocardial ischemia-reperfusion injury in diabetic rats. J Diabetes Investig 2020;11:1126-36.

19. Yamamoto M, Kensler TW, Motohashi H. The KEAP1NRF2 System: a Thiol-Based Sensor-Effector Apparatus for Maintaining Redox Homeostasis. Physiol Rev 2018;98:1169-203.

20. Tonelli C, Chio IIC, Tuveson DA. Transcriptional Regulation by Nrf2. Antioxid Redox Signal 2018;29:1727-45.

21. Hayes JD, Dinkova-Kostova AT. The Nrf2 regulatory network provides an interface between redox and intermediary metabolism. Trends Biochem Sci 2014;39:199-218.

22. Ma Q. Role of nrf2 in oxidative stress and toxicity. Annu Rev Pharmacol Toxicol 2013;53:401-26.

23. Lee DH, Park JS, Lee YS, et al. SQSTM1/p62 activates NFE2L2/NRF2 via ULK1-mediated autophagic KEAP1 degradation and protects mouse liver from lipotoxicity.
Autophagy 2020;16:1949-73.

24. Rao J, Qian X, Li G, et al. ATF3-mediated NRF2/HO-1 signaling regulates TLR4 innate immune responses in mouse liver ischemia/reperfusion injury. Am J Transplant 2015;15:76-87.

25. Zhou Y, Zhang J, Lei B, et al. DADLE improves hepatic ischemia/reperfusion injury in mice via activation of the Nrf2/HO-1 pathway. Mol Med Rep 2017;16:6214-21.

26. Ge M, Yao W, Yuan D, et al. Brg1-mediated Nrf2/HO-1 pathway activation alleviates hepatic ischemia-reperfusion injury. Cell Death Dis 2017;8:e2841.

27. Zhang Q, Lai Y, Deng J, et al. Vagus Nerve Stimulation Attenuates Hepatic Ischemia/Reperfusion Injury via the Nrf2/HO-1 Pathway. Oxid Med Cell Longev 2019;2019:9549506.

28. Engelmann NJ, Clinton SK, Erdman JW Jr. Nutritional aspects of phytoene and phytofluene, carotenoid precursors to lycopene. Adv Nutr 2011;2:51-61.

29. Boyacioglu M, Kum C, Sekkin S, et al. The effects of lycopene on DNA damage and oxidative stress on indomethacin-induced gastric ulcer in rats. Clin Nutr 2016;35:428-35.

30. Phan MAT, Bucknall MP, Arcot J. Interferences of anthocyanins with the uptake of lycopene in Caco2 cells, and their interactive effects on anti-oxidation and anti-inflammation in vitro and ex vivo. Food Chem 2019;276:402-9.

31. Lindsey H. Consuming tomato products may reduce prostate-cancer risk. Lancet Oncol 2002;3:198.

32. Senkus KE, Tan L, Crowe-White KM. Lycopene and Metabolic Syndrome: A Systematic Review of the Literature. Adv Nutr 2019;10:19-29.

33. Zhao Y, Xin Z, Li N, et al. Nano-liposomes of lycopene reduces ischemic brain damage in rodents by regulating iron metabolism. Free Radic Biol Med 2018;124:1-11.

34. Yue R, Xia X, Jiang J, et al. Mitochondrial DNA oxidative damage contributes to cardiomyocyte ischemia/ reperfusion-injury in rats: cardioprotective role of lycopene. J Cell Physiol 2015;230:2128-41.

35. Bayramoglu G, Bayramoglu A, Altuner Y, et al. The effects of lycopene on hepatic ischemia/reperfusion injury in rats. Cytotechnology 2015;67:487-91.

36. Pektaş A, Gemalmaz H, Balkaya M, et al. The shortterm protective effects of lycopene on renal ischemiareperfusion injury in rats. Turk J Urol 2014;40:46-51.

37. Shen XD, Ke B, Zhai Y, et al. CD154-CD40 T-cell costimulation pathway is required in the mechanism of hepatic ischemia/reperfusion injury, and its blockade 
facilitates and depends on heme oxygenase-1 mediated cytoprotection. Transplantation 2002;74:315-9.

38. Boeira SP, Filho CB, Del'Fabbro L, et al. Lycopene treatment prevents hematological, reproductive and histopathological damage induced by acute zearalenone administration in male Swiss mice. Exp Toxicol Pathol 2014;66:179-85.

39. Yue S, Zhu J, Zhang M, et al. The myeloid heat shock transcription factor $1 / \beta$-catenin axis regulates NLR family, pyrin domain-containing 3 inflammasome activation in mouse liver ischemia/reperfusion injury. Hepatology 2016;64:1683-98.

40. Suzuki S, Toledo-Pereyra LH, Rodriguez FJ, et al. Neutrophil infiltration as an important factor in liver ischemia and reperfusion injury. Modulating effects of FK506 and cyclosporine. Transplantation 1993;55:1265-72.

41. Yue S, Rao J, Zhu J, et al. Myeloid PTEN deficiency protects livers from ischemia reperfusion injury by facilitating M2 macrophage differentiation. J Immunol 2014;192:5343-53.

42. Abass MA, Elkhateeb SA, Abd El-Baset SA, et al. Lycopene ameliorates atrazine-induced oxidative damage in adrenal cortex of male rats by activation of the Nrf2/HO-1 pathway. Environ Sci Pollut Res Int 2016;23:15262-74.

43. Dai C, Tang S, Deng S, et al. Lycopene attenuates colistin-induced nephrotoxicity in mice via activation of the Nrf2/HO-1 pathway. Antimicrob Agents Chemother 2015;59:579-85.

44. Winchell CG, Dragan AL, Brann KR, et al. Coxiella burnetii Subverts p62/Sequestosome 1 and Activates Nrf2 Signaling in Human Macrophages. Infect Immun 2018;86:e00608-17.

45. Xu D, Chen L, Chen X, et al. The triterpenoid CDDOimidazolide ameliorates mouse liver ischemia-reperfusion injury through activating the $\mathrm{Nrf} 2 / \mathrm{HO}-1$ pathway enhanced autophagy. Cell Death Dis 2017;8:e2983.

46. Lu L, Yue S, Jiang L, et al. Myeloid Notch1 deficiency activates the RhoA/ROCK pathway and aggravates hepatocellular damage in mouse ischemic livers. Hepatology 2018;67:1041-55.

47. Wan J, Kuang G, Zhang L, et al. Hesperetin attenuated acetaminophen-induced hepatotoxicity by inhibiting hepatocyte necrosis and apoptosis, oxidative stress and inflammatory response via upregulation of heme oxygenase-1 expression. Int Immunopharmacol 2020;83:106435.

48. Dery KJ, Nakamura K, Kadono K, et al. Human Antigen $\mathrm{R}$ (HuR): A New Regulator of Heme Oxygenase-1
Cytoprotection in Mouse and Human Liver Transplant Injury. Hepatology 2020;72:1056-72.

49. Howard TK, Klintmalm GB, Cofer JB, et al. The influence of preservation injury on rejection in the hepatic transplant recipient. Transplantation 1990;49:103-7.

50. Panel M, Ruiz I, Brillet R, et al. Small-Molecule Inhibitors of Cyclophilins Block Opening of the Mitochondrial Permeability Transition Pore and Protect Mice From Hepatic Ischemia/Reperfusion Injury. Gastroenterology 2019;157:1368-82.

51. Motiño O, Francés DE, Casanova N, et al. Protective Role of Hepatocyte Cyclooxygenase-2 Expression Against Liver Ischemia-Reperfusion Injury in Mice. Hepatology 2019;70:650-65.

52. Yang H, Zhou H, Zhuang L, et al. Plasma membranebound $\mathrm{G}$ protein-coupled bile acid receptor attenuates liver ischemia/reperfusion injury via the inhibition of toll-like receptor 4 signaling in mice. Liver Transpl 2017;23:63-74.

53. Zigmond E, Samia-Grinberg S, Pasmanik-Chor M, et al. Infiltrating monocyte-derived macrophages and resident kupffer cells display different ontogeny and functions in acute liver injury. J Immunol 2014;193:344-53.

54. Zhang C, Feng J, Du J, et al. Macrophage-derived IL$1 \alpha$ promotes sterile inflammation in a mouse model of acetaminophen hepatotoxicity. Cell Mol Immunol 2018;15:973-82.

55. Zheng L, Ling W, Zhu D, et al. Roquin-1 Regulates Macrophage Immune Response and Participates in Hepatic Ischemia-Reperfusion Injury. J Immunol 2020;204:1322-33.

56. Chen G, Ni Y, Nagata N, et al. Lycopene Alleviates Obesity-Induced Inflammation and Insulin Resistance by Regulating M1/M2 Status of Macrophages. Mol Nutr Food Res 2019;63:e1900602.

57. Guo H, Callaway JB, Ting JP. Inflammasomes: mechanism of action, role in disease, and therapeutics. Nat Med 2015;21:677-87.

58. Gong Z, Pan J, Shen Q, et al. Mitochondrial dysfunction induces NLRP3 inflammasome activation during cerebral ischemia/reperfusion injury. J Neuroinflammation 2018;15:242.

59. Toldo S, Marchetti C, Mauro AG, et al. Inhibition of the NLRP3 inflammasome limits the inflammatory injury following myocardial ischemia-reperfusion in the mouse. Int J Cardiol 2016;209:215-20.

60. Jin Y, Li C, Xu D, et al. Jagged1-mediated myeloid Notch1 signaling activates HSF1/Snail and controls NLRP3 inflammasome activation in liver inflammatory injury. Cell 
Mol Immunol 2020;17:1245-56.

61. Xu Y, Tang Y, Lu J, et al. PINK1-mediated mitophagy protects against hepatic ischemia/reperfusion injury by restraining NLRP3 inflammasome activation. Free Radic Biol Med 2020;160:871-86.

62. Ueno T, Komatsu M. Autophagy in the liver: functions in health and disease. Nat Rev Gastroenterol Hepatol 2017;14:170-84.

63. Ilyas G, Zhao E, Liu K, et al. Macrophage autophagy limits acute toxic liver injury in mice through down regulation of interleukin-1 $\beta$. J Hepatol 2016;64:118-27.

64. Liu W, Bai F, Wang H, et al. Tim-4 Inhibits NLRP3 Inflammasome via the LKB1/AMPK $\alpha$ Pathway in Macrophages. J Immunol 2019;203:990-1000.

Cite this article as: Xue R, Qiu J, Wei S, Liu M, Wang Q, Wang P, Sha B, Wang H, Shi Y, Zhou J, Rao J, Lu L. Lycopene alleviates hepatic ischemia reperfusion injury via the Nrf2/ HO-1 pathway mediated NLRP3 inflammasome inhibition in Kupffer cells. Ann Transl Med 2021;9(8):631. doi: 10.21037/atm20-7084
65. Zhou S, Gu J, Liu R, et al. Spermine Alleviates Acute Liver Injury by Inhibiting Liver-Resident Macrophage Pro-Inflammatory Response Through ATG5-Dependent Autophagy. Front Immunol 2018;9:948.

66. Kim SH, Kim G, Han DH, et al. Ezetimibe ameliorates steatohepatitis via AMP activated protein kinaseTFEB-mediated activation of autophagy and NLRP3 inflammasome inhibition. Autophagy 2017;13:1767-81.

67. Lee SJ, Ryter SW, Xu JF, et al. Carbon monoxide activates autophagy via mitochondrial reactive oxygen species formation. Am J Respir Cell Mol Biol 2011;45:867-73.

68. Ryter SW. Heme oxygenase-1/carbon monoxide as modulators of autophagy and inflammation. Arch Biochem Biophys 2019;678:108186. 
Primer sequences for the amplification:

\begin{tabular}{cll}
\hline Gene & \multicolumn{1}{c}{ Forward primer $\left(5^{\prime} \rightarrow 3^{\prime}\right)$} & \multicolumn{1}{c}{ Reverse primer $\left(5^{\prime} \rightarrow 3^{\prime}\right)$} \\
\hline TNF- $\alpha$ & $5^{\prime}$-GCCTCTTCTCATTCCTGCTTGT-3' & 5'-GATGATCTGAGTGTGAGGGTCTG-3' \\
IL-13 & $5^{\prime}$-GCAACTGTTCCTGAACTCAACT-3 & 5'-ATCTITTGGGTCCGTCAACT-3' \\
IL-6 & $5^{\prime}$-GCTACCAAACTGGATATAATCAGGA-3' & 5'-CCAGGTAGCTATGGTACTCCAGAA-3' \\
IL-10 & 5'-GCTCTTACTGACTGGCATGAG-3' & 5'-CGCAGCTCTAGGAGCATGTG-3' \\
NLRP3 & 5'-ATTACCCGCCCGAGAAAGG-3' & 5'-TCGCAGCAAAGATCCACACAG-3' \\
GAPDH & 5'-CCATCTTCCAGGAGCGAGATC-3' & 5'-GCCTTCTCCATGGTGGTGAA-3' \\
\hline
\end{tabular}

Figure S1 Primer sequences for the amplification. 